\title{
Godunov-type Numerical Scheme for the Shallow Water Equations with Horizontal Temperature Gradient
}

\author{
Nguyen Xuan Thanh, Mai Duc Thanh* and Dao Huy Cuong
}

\begin{abstract}
We present a Godunov-type scheme for the shallow water equations with horizontal temperature gradient and variable topography. First, the exact solutions of the Riemann problem in a computational form are given, where algorithms for computing these solutions are described. Second, a Godunov-type scheme is constructed relying on exact solutions of the local Riemann problems. Computing algorithms for the scheme are given. The scheme is shown to be well-balanced and preserve the positivity of the water height. Numerical tests show that the scheme is convergent with a good accuracy, even for the resonant phenomenon, where the exact solutions contain several distinct waves propagating with the same shock speed. Furthermore, the scheme also provides us with good results for the solution of the wave interaction problem.
\end{abstract}

\section{Introduction}

In this paper we will construct a Godunov-type scheme for the one-dimensional shallow water equations with variable topography and horizontal temperature gradient (the Ripa system, see [23,24]). The Ripa system was used to model ocean currents, and was derived from the Saint-Venant system of shallow water equations, in which the horizontal water temperature fluctuations are taken into account. Precisely, the governing equations are given by

$$
\partial_{t} h+\partial_{x}(h u)=0, \quad \partial_{t}(h u)+\partial_{x}\left(h u^{2}+\frac{g h^{2} \theta}{2}\right)=-g h \theta \partial_{x} a, \quad \partial_{t}(h \theta)+\partial_{x}(h u \theta)=0
$$

where $h=h(x, t), u=u(x, t)$, and $\theta=\theta(x, t)$ denote the water depth, the depth-averaged horizontal velocity, and the potential temperature field, respectively; $g$ is the gravitational constant, and $a=a(x)$ is the bottom topography.

Observe that the right-hand side of the system (1.1) contains a nonconservative term. In general, nonconservativeness in system of balance laws, including multi-phase flow Received December 23, 2018; Accepted May 1, 2019.

Communicated by Suh-Yuh Yang.

2010 Mathematics Subject Classification. 35L65, 65M08, 76B15.

Key words and phrases. shallow water equations with temperature, topography, nonconservative, Godunov scheme, accuracy, resonant.

*Corresponding author. 
models, often causes lots of inconvenience for standard numerical schemes. For example, oscillations can be produced immediately, or the errors may become larger when the mesh sizes get smaller. Numerical approximations for nonconservative systems have therefore attracted many authors.

Fortunately, supplementing (1.1) with the trivial equation

$$
\partial_{t} a=0 .
$$

one can obtain a system of balance laws in nonconservative form

$$
\partial_{t} U+A(U) \partial_{x} U=0 \text {. }
$$

Therefore, basic properties of the system, and furthermore, the Riemann problem can be studied, see 31. Motivated by the later work, we will construct in this paper a Godunovtype scheme for (1.1). The scheme is based on exact Riemann solvers for different types of configurations of Riemann solutions. Recall that the Riemann problem for (1.1)-(1.2) is the Cauchy problem where the initial data are piecewise constant and are of the form

$$
(h, u, \theta, a)(x, 0)= \begin{cases}\left(h_{L}, u_{L}, \theta_{L}, a_{L}\right) & \text { if } x<0, \\ \left(h_{R}, u_{R}, \theta_{R}, a_{R}\right) & \text { if } x>0 .\end{cases}
$$

In this work, we will first develop the argument in 31 and deliver explicit constructions of exact Riemann solvers in computational form for the system $1.1-(1.2)$ in both resonant and non-resonant regions. That is, left-hand and right-hand states of elementary waves in solutions of the Riemann problem are constraint to nonlinear algebraic equations. Computing roots of these nonlinear algebraic equations by a suitable numerical method will provide us with computational Riemann solutions. These Riemann solvers then provide us with solutions to local Riemann problems which we can use to design a Godunov-type scheme. Then, we prove that the scheme possesses very interesting properties: first, it is well-balanced in the sense that it can capture exactly stationary waves; second, it preserves the positivity of the water height. Numerical experiments are conducted for both kinds of subcritical and supercritical regions. The errors are computed for different mesh sizes. These results show that the scheme is convergent in all the cases of subcritical or supercritical regions. The scheme still converges in the resonant cases, where the exact solution contain multiple waves propagating with the same shock speed. Furthermore, a wave interaction problem is investigated, and the Godunov-type scheme gives us good approximations of the solution of the wave interaction problem.

We note that numerical approximations for nonconservative systems of balance laws have been attractive for many authors. Numerical schemes for a single conservation law with a nonconservative source term were studied in $[3,5,6]$. Various numerical schemes 
for shallow water equations with variable topography and nonconservative systems were proposed in $[8,12,14,16,20,22,25]$. Godunov-type schemes for multi-phase flow models and other hyperbolic systems of balance laws in nonconservative forms are considered in $[2,10,19,27]$. Several numerical schemes for the Ripa system were constructed in 7, 15, 26, 32, 33. See also the references therein.

Well-balanced schemes for the model of a fluid flow in a nozzle with variable crosssection were constructed in [18. Numerical schemes for two-phase flow models were presented in [1,4,9,28. The Riemann problem for other hyperbolic systems in nonconservative form were considered in 17,29,30. See also the references therein.

This paper is organized as follows. In Section 2, we recall basic properties of the system (1.1)-1.2. Then, computing algorithms for stationary contact waves are given. In Section 3, computational forms of the exact solutions of the Riemann problem are provided. Section 4 is devoted to the construction of the Godunonv-type scheme for (1.1). Properties of the scheme are also established. In Section 5 we present numerical tests. Finally, several conclusions and discussions are given in Section 6 .

\section{Preliminaries}

\subsection{Wave curves}

For smooth solutions, the system (1.1)-(1.2) can be re-written as a nonconservative system as

$$
\partial_{t} U+A(U) \partial_{x} U=0
$$

where

$$
U=\left(\begin{array}{l}
h \\
u \\
\theta \\
a
\end{array}\right), \quad A(U)=\left(\begin{array}{cccc}
u & h & 0 & 0 \\
g \theta & u & g h / 2 & g \theta \\
0 & 0 & u & 0 \\
0 & 0 & 0 & 0
\end{array}\right)
$$

The matrix $A(U)$ has four real eigenvalues

$$
\lambda_{1}(U)=u-c, \quad \lambda_{2}(U)=u, \quad \lambda_{3}(U)=u+c, \quad \lambda_{4}(U)=0,
$$

together with the corresponding right eigenvectors which can be chosen as

$$
r_{1}(U)=\left(\begin{array}{c}
h \\
-c \\
0 \\
0
\end{array}\right), \quad r_{2}(U)=\left(\begin{array}{c}
h \\
0 \\
-2 \theta \\
0
\end{array}\right), \quad r_{3}(U)=\left(\begin{array}{l}
h \\
c \\
0 \\
0
\end{array}\right), \quad r_{4}(U)=\left(\begin{array}{c}
c^{2} \\
-g u \theta \\
0 \\
u^{2}-c^{2}
\end{array}\right) \text {, }
$$


where $c=\sqrt{g h \theta}$. The system is strictly hyperbolic on the sets $G_{1}, G_{2}, G_{3}$ and $G_{4}$, where

$$
\begin{array}{ll}
G_{1}=\left\{U: \lambda_{4}(U)<\lambda_{1}(U)\right\}, & G_{2}=\left\{U: \lambda_{1}(U)<\lambda_{4}(U)<\lambda_{2}(U)\right\}, \\
G_{3}=\left\{U: \lambda_{2}(U)<\lambda_{4}(U)<\lambda_{3}(U)\right\}, & G_{4}=\left\{U: \lambda_{3}(U)<\lambda_{4}(U)\right\} .
\end{array}
$$

The first and the fourth characteristic speeds $\lambda_{1}(U)$ and $\lambda_{4}(U)$ coincide on the surface

$$
\mathcal{C}^{+}=\left\{U: \lambda_{1}(U)=\lambda_{4}(U)\right\}
$$

The second and the fourth characteristic speeds $\lambda_{2}(U)$ and $\lambda_{4}(U)$ coincide on the surface

$$
\mathcal{C}^{0}=\left\{U: \lambda_{2}(U)=\lambda_{4}(U)\right\}
$$

The third and the fourth characteristic speeds $\lambda_{3}(U)$ and $\lambda_{4}(U)$ coincide on the surface

$$
\mathcal{C}^{-}=\left\{U: \lambda_{3}(U)=\lambda_{4}(U)\right\}
$$

Let us define the generalized Froude number

$$
\operatorname{Fr}(U)=\frac{|u|}{c}
$$

This means that a supercritical state is the one at which $\operatorname{Fr}(U)>1$; a subcritical state is the one at which $\operatorname{Fr}(U)<1$; and a critical state is the one at which $\operatorname{Fr}(U)=1$.

It is easy to see that the first and third characteristic fields $\left(\lambda_{1}, r_{1}\right),\left(\lambda_{3}, r_{3}\right)$ are genuinely nonlinear, since

$$
-\nabla \lambda_{1} \cdot r_{1}=\nabla \lambda_{3} \cdot r_{3}=\frac{3 c}{2}
$$

and that the second and fourth characteristic fields $\left(\lambda_{2}, r_{2}\right),\left(\lambda_{4}, r_{4}\right)$ are linearly degenerate, since

$$
\nabla \lambda_{2} \cdot r_{2}=\nabla \lambda_{2} \cdot r_{4}=0 .
$$

Let us recall that a discontinuity wave of $[1.1-(1.2)$ is a weak solution of the form

$$
U(x, t)= \begin{cases}U_{-} & \text {if } x<\sigma t, \\ U_{+} & \text {if } x>\sigma t,\end{cases}
$$

where $U_{-}, U_{+}$are the left-hand and right-hand states, respectively, and $\sigma=\sigma\left(U_{-}, U_{+}\right)$is the speed of the discontinuity wave.

The Rankine-Hugoniot relation associated with 1.2 takes the form

$$
-\sigma[a]=0
$$

where $\sigma$ denotes the speed of the discontinuity wave, $[a]=a_{+}-a_{-}$is the jump of the quantity $a$ across the discontinuity wave. Let us fix a left-hand state $U_{-}=U_{0}=\left(h_{0}, u_{0}, \theta_{0}, a_{0}\right)$, and look for all right-hand states $U=U_{+}$of the shock, as discussed in [31], across a discontinuity wave there are two possibilities: 
(i) either the bottom topography a remains constant or

(ii) the speed $\sigma=0=\lambda_{4}\left(U_{ \pm}\right)$, so this is the 4-contact discontinuity wave, so called the stationary contact discontinuity, since this wave is independent of time.

Let us consider the first case (i), where the system $1.1-1.2$ is reduced to the usual shallow water equations with flat bottom

$$
\partial_{t} h+\partial_{x}(h u)=0, \quad \partial_{t}(h u)+\partial_{x}\left(h u^{2}+\frac{g h^{2} \theta}{2}\right)=0, \quad \partial_{t}(h \theta)+\partial_{x}(h u \theta)=0 .
$$

The Rankine-Hugoniot relations associated with 2.5 are given by

$$
-\sigma[h]+[h u]=0, \quad-\sigma[h u]+\left[h u^{2}+\frac{g h^{2} \theta}{2}\right]=0, \quad-\sigma[h \theta]+[h u \theta]=0 .
$$

These above equations yield

$$
[\theta]\left(u_{0}-\sigma\right)=0
$$

which means that either $\theta=\theta_{0}=$ constant, or $\sigma=u_{0}$.

Consider first the case $\theta=\theta_{0}=$ constant. A shock wave 2.3 is the $i$ th-Lax shock $(i=1,3)$ if it satisfies the Lax shock inequalities

$$
\lambda_{i}\left(U_{+}\right)<\sigma\left(U_{-}, U_{+}\right)<\lambda_{i}\left(U_{-}\right), \quad i=1,3 .
$$

Given a left-hand state $U_{0}$, the set of all right-hand states that can be connected to $U_{0}$ by 1 st-Lax shock forms a curve, denoted by $\mathcal{S}_{1}\left(U_{0}\right)$,

$$
\mathcal{S}_{1}\left(U_{0}\right): a=a_{0}, \quad \theta=\theta_{0}, \quad u=u_{0}-\sqrt{\frac{g \theta_{0}}{2}}\left(h-h_{0}\right) \sqrt{\frac{1}{h}+\frac{1}{h_{0}}}, \quad h>h_{0} .
$$

In a backward way, given a right-hand state $U_{0}$, the set of all left-hand states that can be connected to $U_{0}$ by 3 rd-Lax shock forms a curve, denoted by $\mathcal{S}_{3}^{B}\left(U_{0}\right)$

$$
\mathcal{S}_{3}^{B}\left(U_{0}\right): a=a_{0}, \quad \theta=\theta_{0}, \quad u=u_{0}+\sqrt{\frac{g \theta_{0}}{2}}\left(h-h_{0}\right) \sqrt{\frac{1}{h}+\frac{1}{h_{0}}}, \quad h>h_{0},
$$

see 31 .

It is interesting that the shock speeds in the non-linear characteristic fields may coincide with the characteristic speed of the linearly degenerate field as stated in the following lemma.

Lemma 2.1. 19, Lemma 2.1] Consider the projection on the (h,u)-plan. To every $U_{L}=\left(h_{L}, u_{L}\right) \in G_{1}$ there exists exactly one point $U^{\#} \in \mathcal{S}_{1}\left(U_{L}\right) \cap G_{2}$ such that the 1 -shock speed $\sigma_{1}\left(U_{L}, U^{\#}\right)=0$. The state $U^{\#}=\left(h^{\#}, u^{\#}\right)$ is defined by

$$
h^{\#}=\frac{-h_{L}+\sqrt{h_{L}^{2}+8 h_{L} u_{L}^{2} /\left(g \theta_{L}\right)}}{2}, \quad u^{\#}=\frac{u_{L} h_{L}}{h^{\#}} .
$$


Moreover, for any $U \in \mathcal{S}_{1}\left(U_{L}\right)$, the shock speed $\sigma_{1}\left(U_{L}, U\right)>0$ if and only if $U$ is located above $U^{\#}$ on $\mathcal{S}_{1}\left(U_{L}\right)$.

Now, let us consider the case $\sigma=u_{0}$. The relations (2.6) yield

$$
\sigma=u_{0}=u=\lambda_{2}\left(U_{0}\right)=\lambda_{2}(U)
$$

so this is the 2-contact discontinuity wave, so called as the material contact discontinuity. Moreover, the curve of 2-contact discontinuities issuing from a given state $U_{0}$ can be parameterized by $h$ as follows:

$$
\mathcal{W}_{2}\left(U_{0}\right): a=a_{0}, \quad u=u_{0}, \quad \theta=\frac{h_{0}^{2} \theta_{0}}{h^{2}}, \quad h>0 .
$$

Next, let us consider rarefaction waves, which are piecewise smooth self-similar solutions of $1.1-1.2$, i.e.,

$$
U(x, t)=V(\xi), \quad \xi=\frac{x}{t}, \quad x \in \mathbb{R}, t>0 .
$$

Substituting this into 2.1, we can see that rarefaction waves are solutions of the following initial-value problem for ordinary differential equations

$$
\frac{d V(\xi)}{d \xi}=r_{j}(V(\xi)), \quad \xi \geq \lambda_{j}\left(U_{0}\right), \quad V\left(\lambda_{j}\left(U_{0}\right)\right)=U_{0}, \quad j=1,3
$$

where the eigenvectors $r_{1}$ and $r_{3}$ are given by (2.2). In particular, it holds along the integral curves that

$$
\frac{d \theta(\xi)}{d \xi}=\frac{d a(\xi)}{d \xi}=0
$$

which means that the temperature and the bottom topography a remain constant through any rarefaction fan.

Furthermore, it holds from 2.7 for $i=1$ that

$$
\frac{d u}{d h}=-\sqrt{\frac{g \theta_{0}}{h}} .
$$

This implies that the integral curve associated with the first characteristic field passing through a given point $U_{0}$ is given by

$$
u=u_{0}-2 \sqrt{g \theta_{0}}\left(\sqrt{h}-\sqrt{h_{0}}\right) .
$$

Since the characteristic speed should be increasing through a rarefaction fan, 1-rarefaction waves correspond to the part $h \geq h_{0}$, on the integral curve. Thus, we can define the forward curve of 1-rarefaction waves $\mathcal{R}_{1}\left(U_{0}\right)$ issuing from a given left-hand state $U_{0}$, which consists 
of all the right-hand states $U$ that can be connected to $U_{0}$ by a rarefaction wave associated with the first characteristic field by

$$
\mathcal{R}_{1}\left(U_{0}\right): u=u_{0}-2 \sqrt{\frac{g \theta_{0}}{2}}\left(\sqrt{h}-\sqrt{h_{0}}\right), \quad h<h_{0}
$$

Similarly, the backward curve of 3-rarefaction waves $\mathcal{R}_{3}^{B}\left(U_{0}\right)$ issuing from a given righthand state $U_{0}$ consisting if all the left-hand states $U$ that can be connected to $U_{0}$ by a rarefaction wave associated with the third characteristic field by

$$
\mathcal{R}_{3}^{B}\left(U_{0}\right): u=u_{0}+2 \sqrt{\frac{g \theta_{0}}{2}}\left(\sqrt{h}-\sqrt{h_{0}}\right), \quad h<h_{0}
$$

From above analysis, we can define the wave curves

$$
\mathcal{W}_{1}\left(U_{0}\right)=\mathcal{S}_{1}\left(U_{0}\right) \cup \mathcal{R}_{1}\left(U_{0}\right), \quad \mathcal{W}_{3}^{B}\left(U_{0}\right)=\mathcal{S}_{3}^{B}\left(U_{0}\right) \cup \mathcal{R}_{3}^{B}\left(U_{0}\right)
$$

From 2.8 , we can show that the curves $\mathcal{W}_{1}\left(U_{0}\right), \mathcal{W}_{3}^{B}\left(U_{0}\right)$ can be parameterized as

$$
\begin{gathered}
\mathcal{W}_{1}\left(U_{0}\right): u=\omega_{1}\left(U_{0}, h\right)= \begin{cases}u_{0}-\sqrt{\frac{g \theta_{0}}{2}}\left(h-h_{0}\right) \sqrt{\frac{1}{h}+\frac{1}{h_{0}}} & \text { if } h>h_{0}, \\
u_{0}-2 \sqrt{\frac{g \theta_{0}}{2}}\left(\sqrt{h}-\sqrt{h_{0}}\right) & \text { if } h<h_{0},\end{cases} \\
\mathcal{W}_{3}^{B}\left(U_{0}\right): u=\omega_{3}^{B}\left(U_{0}, h\right)= \begin{cases}u_{0}+\sqrt{\frac{g \theta_{0}}{2}}\left(h-h_{0}\right) \sqrt{\frac{1}{h}+\frac{1}{h_{0}}} & \text { if } h>h_{0}, \\
u_{0}+2 \sqrt{\frac{g \theta_{0}}{2}}\left(\sqrt{h}-\sqrt{h_{0}}\right) & \text { if } h<h_{0} .\end{cases}
\end{gathered}
$$

Suppose that $U \in \mathcal{W}_{3 \leftarrow 2}\left(U_{R}\right)$ implies that consists of all left-hand state $U$ which can be reached from $U_{R}$ using a combination of a 3 -wave from right-hand state $U_{R}$ to some left-hand state $U_{M}$, preceded by a 2-wave from the right-hand state $U_{M}$ to the left-hand state $U, U_{M}=\mathcal{W}_{2}(U) \cap \mathcal{W}_{3}^{B}\left(U_{R}\right)$, where

$$
U=(h, u, \theta, a), \quad U_{M}=\left(h_{M}, u_{M}, \theta_{M}, a_{M}\right),
$$

we have $u=u_{M}=\omega_{3}^{B}\left(U_{R}, h\right)$ because $U$ connects to $U_{M}$ by 2-contact discontinuities.

Accordingly, for each $U=(h, u, \theta, a)$, we define $\phi_{3 \leftarrow 2}\left(U_{R}, U\right)$ as follows:

$$
\phi_{3 \leftarrow 2}\left(U_{R}, U\right)=u-\omega_{3}^{B}\left(U_{R}, h\right),
$$

where the function $\omega_{3}^{B}\left(U_{R}, h\right)$ is defined as in 2.9$)$. Obviously, $\phi_{3 \leftarrow 2}\left(U_{R}, U\right)=0$ for $U \in$ $\mathcal{W}_{3 \leftarrow 2}\left(U_{R}\right)$. Moreover, $\phi_{3 \leftarrow 2}\left(U_{R}, U\right)>0$ for $U$ is above $\mathcal{W}_{3 \leftarrow 2}\left(U_{R}\right)$ and $\phi_{3 \leftarrow 2}\left(U_{R}, U\right)<0$ for $U$ is below $\mathcal{W}_{3 \leftarrow 2}\left(U_{R}\right)$. 


\subsection{Stationary contact discontinuities}

In this subsection we will define a new kind of waves, called stationary contact waves, corresponding to the case (ii) of (2.4). They are weak solutions of (1.1) that are independent of time. To see that they are weak solutions, we first consider stationary smooth solutions of (1.1), which are independent of time. Then, the system (1.1) for smooth stationary solutions becomes

$$
(h u)^{\prime}=0, \quad\left(h u^{2}+\frac{g}{2} h^{2} \theta\right)^{\prime}+g h \theta a^{\prime}=0, \quad(h u \theta)^{\prime}=0,
$$

where $(\cdot)^{\prime}=d(\cdot) / d x$.

Theorem 2.2. (a) Any solution of the following system of ordinary differential equations

$$
(h u)^{\prime}=0, \quad\left(\frac{u^{2}}{2}+g \theta(h+a)\right)^{\prime}=0, \quad \theta^{\prime}=0
$$

is a stationary smooth solution of 1.1 .

(b) Any discontinuity 2.3 with speed $\sigma=0$ satisfying the jump relations

$$
[h u]=0, \quad\left[\frac{u^{2}}{2}+g \theta(h+a)\right]=0, \quad[\theta]=0
$$

is a weak solution of 1.1 in sense of non-conservative product corresponding the path $s \in[0,1] \mapsto \phi(s):=(h(s), u(s), \theta(s), a(s))$ defined by 2.10 such that $\phi(0)=$ $\left(h_{-}, u_{-}, \theta_{-}, a_{-}\right)$and $\phi(1)=\left(h_{+}, u_{+}, \theta_{+}, a_{+}\right)$, see [11].

(c) The integral curve associated with the characteristic field $\left(\lambda_{4}, r_{4}\right)$ is a solution of 2.10 .

Proof. (a) From the 1st equation and the 3rd equation of 2.10, we have

$$
(h u \theta)^{\prime}=(h u)^{\prime} \theta+(h u) \theta^{\prime}=0 .
$$

From the 2nd equation, we obtain

$$
\left(h u^{2}+\frac{g}{2} h^{2} \theta\right)^{\prime}+g h \theta a^{\prime}=(h u) u^{\prime}+g \theta h h^{\prime}+g h \theta a^{\prime}=h\left(\frac{u^{2}}{2}+g \theta(h+a)\right)^{\prime}=0 .
$$

Thus, any solution of 2.10 is a stationary smooth solution of 1.1 .

(b) Consider the functions $U^{\varepsilon}$ defined by

$$
U^{\varepsilon}(x, t)= \begin{cases}U_{-} & \text {if } x<-\varepsilon \\ \phi\left(\frac{x+\varepsilon}{2 \varepsilon}\right) & \text { if }-\varepsilon \leq x \leq \varepsilon \\ U_{+} & \text {if } x>\varepsilon\end{cases}
$$


It is obvious that $U^{\varepsilon}$ are weak solutions of (1.1) and converge almost everywhere to the discontinuity (2.3) with speed $\sigma=0$, when $\varepsilon \rightarrow 0^{+}$. By the stability result of nonconservative product, we imply that any the discontinuity 2.3 with speed $\sigma=0$ satisfying the jump relations (2.11) is a weak solution of (1.1) in sense non-conservative product, see 11 .

(c) Consider the integral curve associated with the characteristic field $\left(\lambda_{4}, r_{4}\right)$, i.e.,

$$
\frac{d U}{d \xi}=r_{4}(U(\xi))
$$

where $U(\xi)=(h(\xi), u(\xi), \theta(\xi), a(\xi))$. From 2.2$)$, we have

$$
h^{\prime}=g h \theta, \quad u^{\prime}=-g u \theta, \quad \theta^{\prime}=0, \quad a^{\prime}=u^{2}-g h \theta .
$$

Therefore, we obtain

$$
(h u)^{\prime}=h^{\prime} u+h u^{\prime}=(g h \theta) u+h(-g u \theta)=0
$$

and

$$
\begin{aligned}
\left(\frac{u^{2}}{2}+g \theta(h+a)\right)^{\prime} & =u u^{\prime}+g \theta^{\prime}(h+a)+g \theta\left(h^{\prime}+a^{\prime}\right) \\
& =u(-g u \theta)+g \theta\left(g h \theta+\left(u^{2}-g h \theta\right)\right) \\
& =0 .
\end{aligned}
$$

Thus, the integral curve associated with the characteristic field $\left(\lambda_{4}, r_{4}\right)$ is a solution of (2.10).

We call the discontinuity 2.3 with speed $\sigma=0$ satisfying the jump relations 2.11 ) the 4-contact wave.

Definition 2.3. The elementary waves of (1.1) are

- $k$-Lax shock waves, $k=1,3$;

- $k$-rarefaction waves, $k=1,3$;

- 2-contact waves;

- 4-contact waves satisfying the jump relations 2.11. 


\subsection{Properties of stationary contact discontinuities}

Given a state $U_{0}$ and bottom levels on both sides of the stationary contact $a \neq a_{0}$, we solve for the water height $h$ in terms of other known quantities from the 2nd equation of (2.11). Multiplying both sides of this equation by $2 g \theta_{0} h^{2}$, and then re-arranging terms, we obtain

$$
\varphi(h)=2 g \theta_{0} h^{3}+\left(2 g \theta_{0}\left(a-a_{0}-h_{0}\right)-u_{0}^{2}\right) h^{2}+h_{0}^{2} u_{0}^{2}=0 .
$$

It holds that

$$
\begin{aligned}
\varphi(0) & =h_{0}^{2} u_{0}^{2} \geq 0, \\
\varphi^{\prime}(h) & =6 g \theta_{0} h+2\left(2 g \theta_{0}\left(a-a_{0}-h_{0}\right)-u_{0}^{2}\right) h, \\
\varphi^{\prime \prime}(h) & =12 g \theta_{0} h+2\left(2 g \theta_{0}\left(a-a_{0}-h_{0}\right)-u_{0}^{2}\right) .
\end{aligned}
$$

Solving

$$
\varphi^{\prime}(h)=0, \quad h>0
$$

gives us a unique critical point of $\varphi$

$$
h=h_{*}=\frac{u_{0}^{2}+2 g \theta_{0}\left(a_{0}+h_{0}-a\right)}{3 g \theta_{0}} .
$$

If $h_{*}<0$, or equivalently,

$$
a>a_{0}+h_{0}+\frac{u_{0}^{2}}{2 g},
$$

then $\varphi^{\prime}(h)>0$ for $h>0$. Since $\varphi(0)=h_{0}^{2} u_{0}^{2}$, 2.12 has two roots $h_{1} \leq h_{2}$, if

$$
\varphi\left(h_{*}\right)=-g h_{*}^{3}+h_{0}^{2} u_{0}^{2} \leq 0,
$$

or

$$
h_{*} \geq h_{m}:=\left(\frac{h_{0}^{2} u_{0}^{2}}{g \theta_{0}}\right)^{1 / 3} .
$$

Moreover, the inequality 2.13 is equivalent to

$$
\begin{aligned}
a \leq a_{\max }\left(U_{0}\right) & :=a_{0}+h_{0}+\frac{u_{0}^{2}}{2 g \theta_{0}}-\frac{3}{2\left(g \theta_{0}\right)^{1 / 3}}\left(h_{0} u_{0}\right)^{2 / 3} \\
& =a_{0}+\frac{1}{2 g \theta_{0}}\left(\left(g \theta_{0} h_{0}\right)^{1 / 3}-u_{0}^{2 / 3}\right)^{2}\left(2\left(g \theta_{0} h_{0}\right)^{1 / 3}+u_{0}^{2 / 3}\right) .
\end{aligned}
$$

The formula 2.14 implies $a_{\max }\left(U_{0}\right) \geq a_{0}$ and the equality holds only if $U_{0}$ belongs to the non-strictly hyperbolic surfaces. Note that if the inequality in 2.14 is strict, i.e., $a<$ $a_{\max }\left(U_{0}\right)$, then the two roots are distinct: $h_{1}(a)<h_{*}<h_{2}(a)$. Consequently, whenever (2.14) holds, there are two states $U_{+}=U_{i}$ so that a stationary contact discontinuity from $U_{-}=U_{0}$ is possible. The locations of these states can be determined in the following lemma: 
Lemma 2.4. 31, Lemma 3.1] Assume that $a<a_{\max }\left(U_{0}\right)$, where $a_{\max }\left(U_{0}\right)$ is defined by (2.14). The function $\varphi$ defined by (2.12) admits two distinct roots $h_{1}<h_{2}$. Moreover, the state $U_{1}$ using the smaller root $h_{1}$ belongs to $G_{1}$ if $u_{0}>0$ and belongs to $G_{4}$ if $u_{0}<0$; the state $U_{2}$ using the larger root $h_{2}$ lies in $G_{2}$ if $u_{0}>0$ and lies in $G_{3}$ if $u_{0}<0$.

To select a unique Riemann solution, the following admissibility criterion for stationary contact discontinuities is imposed:

(MC) Along any stationary curve $\mathcal{W}_{4}\left(U_{0}\right)$, the bottom level $a$ is monotone as a function of $h$. The total variation of the bottom level component of any Riemann solution must not exceed $\left|a_{L}-a_{R}\right|$, where $a_{L}, a_{R}$ are left-hand and right-hand bottom levels.

A similar criterion was used [17, 19].

Lemma 2.5. [31, Lemma 3.2] Assume that $a<a_{\max }\left(U_{0}\right)$, where $a_{\max }\left(U_{0}\right)$ is defined by 2.14. The Monotonicity Criterion selects the following admissible stationary contact wave.

(i) If $U_{0} \in G_{1} \cup G_{4}$, then only the stationary contact using the smaller root $h_{1}$ of $\varphi$ defined by 2.12) is selected.

(ii) If $U_{0} \in G_{2} \cup G_{3}$, then only the stationary contact using the larger root $h_{2}$ of $\varphi$ defined by 2.12 is selected.

We therefore define elementary waves as follows:

Definition 2.6. 31, Definition 3.3] The admissible elementary waves for the system (1.1) and 1.2 are the following ones: Shock waves, rarefaction waves, material conact discontinuities, and admissible stationary contact discontinuities.

\section{The Riemann problem revisited}

Observe that by the transformation $x \mapsto-x, u \mapsto-u$, a left-hand (right-hand) state $U=$ $(h, u, \theta, a)$ in $G_{3}$ (in $G_{4} \cup \mathcal{C}^{-}$) will be transformed to the right-hand (left-hand, respectively) state $V=(h,-u, \theta, a)$ in $G_{2}$ (in $G_{1} \cup \mathcal{C}^{+}$, respectively). Thus, the construction of wave curves and therefore the Riemann solutions for Riemann data around $\mathcal{C}^{-}$can be obtained from the one for Riemann data around $\mathcal{C}^{+}$. Thus, without loss of generality, in the sequel we consider only the case where Riemann data are in $G_{1} \cup \mathcal{C}^{+} \cup G_{2}$.

Notations. We will use the following notations in the sequel:

(i) $\mathcal{W}_{i}\left(U_{1}, U_{2}\right)$ : An $i$-wave connecting a state $U_{1}$ and a state $U_{2}$, where $\mathcal{W}=S$ : Lax shock, $\mathcal{W}=R$ : rarefaction wave, $\mathcal{W}_{2}=Z, i=1,2,3,4$; 
(ii) $\mathcal{W}_{i}\left(U_{1}, U_{2}\right) \rightarrow \mathcal{W}_{j}\left(U_{2}, U_{3}\right)$ : an $i$ wave from the left-hand state $U_{1}$ to the right-hand state $U_{2}$ is followed by a $j$-wave from the left-hand state $U_{2}$ to the right-hand state $u_{3}$;

(iii) $\mathcal{W}_{i}\left(U_{1}, U_{2}\right) \leftarrow \mathcal{W}_{j}\left(U_{2}, U_{3}\right)$ : an $i$ wave from the right-hand state $u_{1}$ to the left-hand state $U_{2}$ is preceded by a $j$-wave from the right-hand state $U_{2}$ to the left-hand state $U_{3}$;

(iv) The (forward) curve $\mathcal{W}_{i \rightarrow j}\left(U_{0}\right)$ consists of all right-hand states $U$ which can be reached from $U_{0}$ using an $i$-wave from the left-hand state $U_{0}$ to some intermediate right-hand state $U_{1} \in \mathcal{W}_{i}\left(U_{0}\right)$, followed by a $j$-wave from the left-hand state $U_{1}$ to the right-hand state $U$;

(v) The (backward) curve $\mathcal{W}_{i \leftarrow j}\left(U_{0}\right)$ consists of all left-hand states $U$ which can be reached from $U_{0}$ using an $i$-wave from the right-hand state $U_{0}$ to some intermediate left-hand state $U_{1} \in \mathcal{W}_{i}\left(U_{0}\right)$, preceded by a $j$-wave from the right-hand state $U_{1}$ to the left-hand state $U$;

(vi) $U^{s}, U^{b}$ denote the states resulted by stationary contact discontinuity wave from $U$;

(vii) $U^{\#}$ denotes the state resulting from a zero-speed shock wave from $U$;

(viii) $U^{ \pm}=\mathcal{W}_{1}\left(U_{L}\right) \cap \mathcal{C}^{ \pm}$

(ix) $U^{0}=\mathcal{W}_{1}\left(U_{L}\right) \cap \mathcal{C}^{0}$.

\subsection{Case A: $U_{L} \in G_{1} \cup \mathcal{C}^{+}$}

In this section we build three constructions A1, A2, and A3. In each construction, $U_{L}$ starts from $G_{1}$.

Construction A1: Supercritical left-hand state and supercritical right-hand state.

First, the solution begins with a stationary wave from $U_{L}$ to the state $U_{1}=U_{L}^{s}$ using smaller root of $\varphi\left(U_{L}, a_{R}\right)=0$ to shift $a_{L}$ to $a_{R}$, where

$$
U_{L}^{s}=\left(h_{L}^{s}, u_{L}^{s}, \theta_{L}, a_{R}\right), \quad h_{L}^{s} \text { is a solution of } \varphi\left(U_{L}, a_{R}\right)=0
$$

Second, we continue with the wave curve $\mathcal{W}_{1}\left(U_{1}\right)$. Let $U_{2} \in \mathcal{W}_{1}\left(U_{1}\right)$ and $U_{2} \in \mathcal{W}_{3 \leftarrow 2}\left(U_{R}\right)$ :

$$
\left\{U_{2}\right\}=\mathcal{W}_{1}\left(U_{1}\right) \cap \mathcal{W}_{3 \leftarrow 2}\left(U_{R}\right),
$$

we can compute $U_{2}$ from the equation

$$
\phi_{3 \leftarrow 2}\left(U_{R}, U_{2}\right)=0,
$$


and get $U_{2}=\left(h_{2}, u_{2}, \theta_{L}, a_{R}\right)$. The equation (3.1) admits a solution when $U_{1}^{\#}$ is below wave curve $\mathcal{W}_{3 \leftarrow 2}\left(U_{R}\right)$ and $U_{1}$ is above wave curve $\mathcal{W}_{3 \leftarrow 2}\left(U_{R}\right)$ or

$$
\phi_{3 \leftarrow 2}\left(U_{R}, U_{1}^{\#}\right) \cdot \phi_{3 \leftarrow 2}\left(U_{R}, U_{1}\right)<0
$$

where we can determine $U_{1}^{\#}=\left(h_{1}^{\#}, u_{1}^{\#}, \theta_{L}, a_{R}\right)$ and $h_{1}^{\#}, u_{1}^{\#}$ are defined as in Lemma 2.1

Third, we can determine $U_{3} \in \mathcal{W}_{2}\left(U_{2}\right)$, so $U_{3}=\left(\sqrt{h_{2}^{2} \theta_{L} / \theta_{R}}, u_{2}, \theta_{R}, a_{R}\right)$. Consequently, the Riemann problem for (1.1) has a solution of the form

$$
\mathcal{W}_{4}\left(U_{L}, U_{1}\right) \rightarrow \mathcal{W}_{1}\left(U_{1}, U_{2}\right) \rightarrow \mathcal{W}_{2}\left(U_{2}, U_{3}\right) \rightarrow \mathcal{W}_{3}\left(U_{3}, U_{R}\right)
$$

See Figure 3.1.

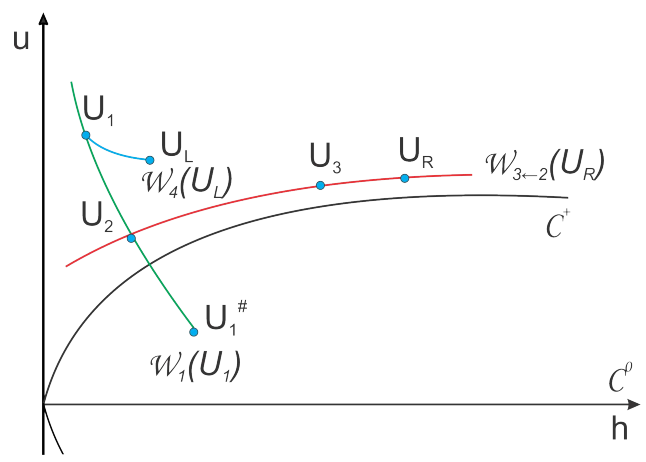

Figure 3.1: A Riemman solution of the form 3.2 in Construction A1.

Remark 3.1. Note that the left-hand state $U_{-}$and right-hand state $U_{+}$of a 2 -contact wave in a Riemann solution are related by

$$
u_{+}=u_{-}, \quad \theta_{R} h_{+}^{2}=\theta_{L} h_{-}^{2} .
$$

Construction A2: Supercritical left-hand state and subcritical right-hand state.

First, the solution begins with 1 -shock wave from $U_{L}$ to a state $U_{1}$, where

$$
U_{1} \in \mathcal{S}_{1}\left(U_{L}\right), \quad \sigma_{1}\left(U_{L}, U_{1}\right) \leq 0
$$

So, $U_{1}$ is located between $U_{L}^{\#}$ and $U^{0}$ on $\mathcal{S}_{1}\left(U_{L}\right)$, where

$$
\begin{aligned}
U_{L}^{\#} & =\left(h_{L}^{\#}, u_{L}^{\#}, \theta_{L}, a_{L}\right) \quad \text { and } h_{L}^{\#}, u_{L}^{\#} \text { are defined as in Lemma 2.1. } \\
U^{0} & =\mathcal{S}_{1}\left(U_{L}\right) \cap \mathcal{C}^{0} .
\end{aligned}
$$

Second, the next part of the solution is a stationary wave from $U_{1}$ to $U_{2}$ to shift $a_{L}$ to $a_{R}$, where

$$
\left\{U_{2}\right\}=\mathcal{W}_{1 \rightarrow 4}\left(U_{L}\right) \cap \mathcal{W}_{3 \leftarrow 2}\left(U_{R}\right)
$$


We solve equation $\phi_{3 \leftarrow 2}\left(U_{R}, U_{2}\right)=0$ to find $h_{1}, U_{1}, U_{2}$, which exists solution when $U_{1}$ is above wave curve $\mathcal{W}_{3 \leftarrow 2}\left(U_{R}\right)$ and $U_{1}^{b}$ is below wave curve $\mathcal{W}_{3 \leftarrow 2}\left(U_{R}\right)$ or

$$
\phi_{3 \leftarrow 2}\left(U_{R}, U_{1}\right) \cdot \phi_{3 \leftarrow 2}\left(U_{R}, U_{1}^{b}\right)<0,
$$

where

$$
\begin{aligned}
& U_{1}=\left(h_{1}, u_{1}, \theta_{L}, a_{L}\right) \in \mathcal{S}_{1}\left(U_{L}\right), \\
& U_{2}=U_{1}^{b}=\left(h_{1}^{b}, u_{1}^{b}, \theta_{L}, a_{R}\right), \quad h_{1}^{b} \text { is the bigger root of } \varphi\left(U_{1}, a_{R}\right)=0 .
\end{aligned}
$$

Third, we can determine $U_{3} \in \mathcal{W}_{2}\left(U_{2}\right)$, so $U_{3}=\left(\sqrt{h_{2}^{2} \theta_{L} / \theta_{R}}, u_{2}, \theta_{R}, a_{R}\right)$. Consequently, the Riemann problem for (1.1) has a solution of the form

$$
\mathcal{W}_{1}\left(U_{L}, U_{1}\right) \rightarrow \mathcal{W}_{4}\left(U_{1}, U_{2}\right) \rightarrow \mathcal{W}_{2}\left(U_{2}, U_{3}\right) \rightarrow \mathcal{W}_{3}\left(U_{3}, U_{R}\right)
$$

See Figure 3.2 .

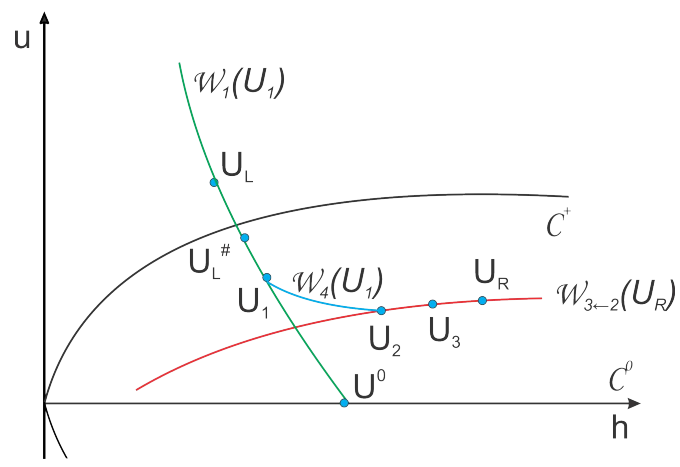

Figure 3.2: A Riemman solution of the form $(3.3)$ in Construction A2.

Construction A3: A resonant case. Supercritical left-hand state and right-hand state near critical curve. In this case, one can see a very interesting phenomenon that the exact solution contains three waves which propagate with the same zero speed. This is the so-called resonant phenomenon.

First, for each bottom high level $a_{1} \in\left[a_{L}, a_{R}\right]$, the solution begins with a stationary wave from $U_{L}$ to $U_{1}$ using smaller root of $\varphi\left(U_{L}, a_{1}\right)=0$ to shift $a_{L}$ to $a_{1}$, where

$$
U_{1}=\left(h_{1}, u_{1}, \theta_{L}, a_{1}\right) \in \mathcal{W}_{4}\left(U_{L}\right), \quad h_{1} \text { is the smaller root of } \varphi\left(U_{L}, a_{1}\right)=0 \text {. }
$$

Second, the next part of the solution is a zero-speed 1-shock from $U_{1}$ to $U_{2}=U_{1}^{\#}$, where $U_{1}^{\#}=\left(h_{1}^{\#}, u_{1}^{\#}, \theta_{L}, a_{1}\right)$ and $h_{1}^{\#}, u_{1}^{\#}$ are defined as in Lemma 2.1.

Third, the next part of the solution is a stationary wave from $U_{2}$ to $U_{3}$ to shift $a_{2}$ to $a_{R}$, where

$$
\left\{U_{3}\right\}=\mathcal{W}_{4 \rightarrow 1 \rightarrow 4}\left(U_{L}\right) \cap \mathcal{W}_{3 \leftarrow 2}\left(U_{R}\right)
$$


We solve the equation $\phi_{3 \leftarrow 2}\left(U_{R}, U_{3}\right)=0$ to find $a_{1}, U_{1}, U_{2}, U_{3}$. This equation admits a solution when $U_{2}^{b}$ is below wave curve $\mathcal{W}_{3 \leftarrow 2}\left(U_{R}\right)$ and $U_{2}$ is above wave curve $\mathcal{W}_{3 \leftarrow 2}\left(U_{R}\right)$. That is,

$$
\phi_{3 \leftarrow 2}\left(U_{R}, U_{2}^{b}\right) \cdot \phi_{3 \leftarrow 2}\left(U_{R}, U_{2}\right)<0,
$$

where

$$
\begin{aligned}
& U_{1}=\left(h_{1}, u_{1}, \theta_{L}, a_{1}\right), \quad U_{2}=U_{1}^{\#}=\left(h_{1}^{\#}, u_{1}^{\#}, \theta_{L}, a_{1}\right), \\
& U_{3}=U_{2}^{b}=\left(h_{2}^{b}, u_{2}^{b}, \theta_{L}, a_{R}\right) \quad \text { when } \phi_{3 \leftarrow 2}\left(U_{R}, U_{2}^{b}\right)=0, \\
& h_{2}^{b} \text { is the bigger root of } \varphi\left(U_{2}, a_{R}\right)=0 .
\end{aligned}
$$

Fourth, we can determine $U_{4} \in \mathcal{W}_{2}\left(U_{3}\right)$, so $U_{4}=\left(\sqrt{h_{3}^{2} \theta_{L} / \theta_{R}}, u_{3}, \theta_{R}, a_{R}\right)$. Consequently, the Riemann problem for 1.1) has a solution of the form

$$
\mathcal{W}_{4}\left(U_{L}, U_{1}\right) \rightarrow \mathcal{W}_{1}\left(U_{1}, U_{2}\right) \rightarrow \mathcal{W}_{4}\left(U_{2}, U_{3}\right) \rightarrow \mathcal{W}_{2}\left(U_{3}, U_{4}\right) \rightarrow \mathcal{W}_{3}\left(U_{4}, U_{R}\right)
$$

See Figure 3.3 .

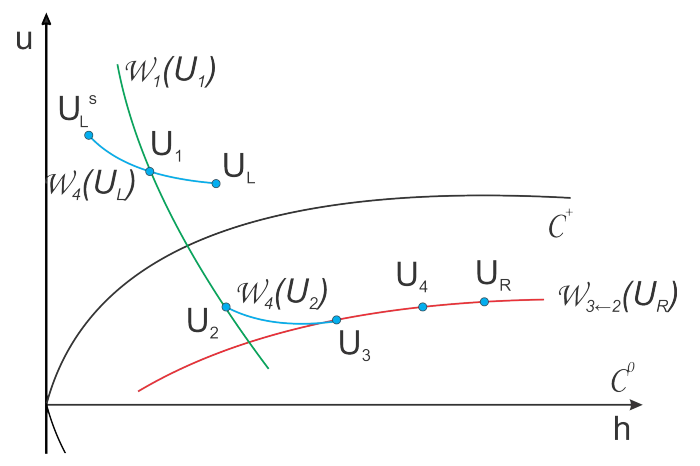

Figure 3.3: A Riemman solution of the form (3.4) in Construction A3.

\subsection{Case B: $U_{L} \in G_{2}$}

Similar to Case A, in this section we build three constructions B1, B2, and B3. But in each construction, $U_{L}$ starts from $G_{2}$.

Construction B1: Subcritical left-hand state and supercritical right-hand state.

First, the solution begins with a 1-rarefaction wave from $U_{L}$ to $U_{1}=U^{+}$, where

$$
U^{+}=\left(h^{+}, u^{+}, \theta_{L}, a_{L}\right)=\mathcal{R}_{1}\left(U_{L}\right) \cap \mathcal{C}^{+} .
$$

Second, the next part of the solution is a stationary wave from $U_{1}$ to $U_{2}=U_{1}^{s}$ using smaller root of $\varphi\left(U_{1}, a_{R}\right)=0$ to shift $a_{1}$ to $a_{R}$, where

$$
U_{1}^{s}=\left(h_{1}^{s}, u_{1}^{s}, \theta_{L}, a_{R}\right) \in \mathcal{W}_{4}\left(U_{1}\right), \quad h_{1}^{s} \text { is the smaller root of } \varphi\left(U_{1}, a_{R}\right)=0 .
$$


Third, the next part of the solution is 1-wave from $U_{2}$ to $U_{3}$, where

$$
\left\{U_{3}\right\}=\mathcal{W}_{1}\left(U_{2}\right) \cap \mathcal{W}_{3 \leftarrow 2}\left(U_{R}\right)
$$

We solve equation $\phi_{3 \leftarrow 2}\left(U_{R}, U_{3}\right)=0$ to find $U_{3}=\left(h_{3}, u_{3}, \theta_{L}, a_{R}\right)$. A solution of this equation exists when $U_{2}^{\#}$ is below wave curve $\mathcal{W}_{3 \leftarrow 2}\left(U_{R}\right)$ and $U_{2}$ is above wave curve $\mathcal{W}_{3 \leftarrow 2}\left(U_{R}\right)$. That yields

$$
\phi_{3 \leftarrow 2}\left(U_{R}, U_{2}^{\#}\right) \cdot \phi_{3 \leftarrow 2}\left(U_{R}, U_{2}\right)<0
$$

where $U_{2}^{\#}=\left(h_{2}^{\#}, u_{2}^{\#}, \theta_{L}, a_{R}\right)$ and $h_{2}^{\#}, u_{2}^{\#}$ are defined as in Lemma 2.1.

Fourth, we can determine $U_{4} \in \mathcal{W}_{2}\left(U_{3}\right)$, so $U_{4}=\left(\sqrt{h_{3}^{2} \theta_{L} / \theta_{R}}, u_{3}, \theta_{R}, a_{R}\right)$. Consequently, the Riemann problem for (1.1) has a solution of the form

$$
\mathcal{W}_{1}\left(U_{L}, U_{1}\right) \rightarrow \mathcal{W}_{4}\left(U_{1}, U_{2}\right) \rightarrow \mathcal{W}_{1}\left(U_{2}, U_{3}\right) \rightarrow \mathcal{W}_{2}\left(U_{3}, U_{4}\right) \rightarrow \mathcal{W}_{3}\left(U_{4}, U_{R}\right)
$$

See Figure 3.4.

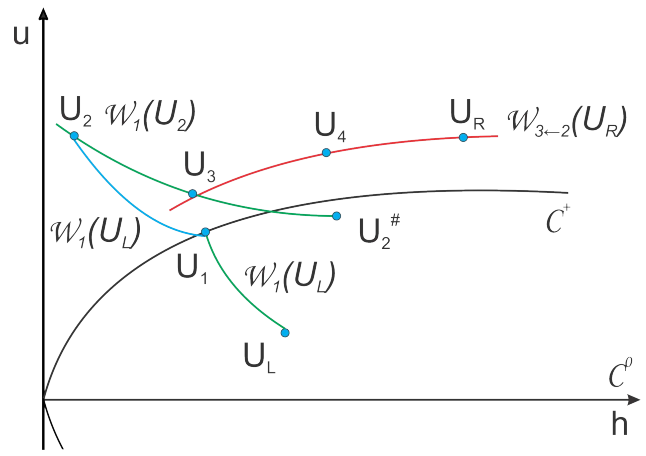

Figure 3.4: A Riemman solution of the form 3.5 in Construction B1.

Construction B2: Subcritical left-hand state and subcritical right-hand state.

First, the solution begins with the 1 -wave from $U_{L}$ to $U_{1}$, where

$$
U_{1}=\left(h_{1}, u_{1}, \theta_{L}, a_{L}\right) \in \mathcal{W}_{1}\left(U_{L}\right)
$$

Obviously, $U_{1}$ is located between $U^{+}$and $U^{0}$ on $\mathcal{W}_{1}\left(U_{L}\right)$, where

$$
U^{+}=\mathcal{W}_{1}\left(U_{L}\right) \cap \mathcal{C}^{+}, \quad U^{0}=\mathcal{W}_{1}\left(U_{L}\right) \cap \mathcal{C}^{0}
$$

Second, the next part of the solution is a stationary wave from $U_{1}$ to $U_{2}$ to shift $a_{L}$ to $a_{R}$, where

$$
\left\{U_{2}\right\}=\mathcal{W}_{1 \rightarrow 4}\left(U_{L}\right) \cap \mathcal{W}_{3 \leftarrow 2}\left(U_{R}\right)
$$


We solve equation $\phi_{3 \leftarrow 2}\left(U_{R}, U_{2}\right)=0$ to find $h_{1}, U_{1}, U_{2}$, which has a solution when $U_{1}$ is above wave curve $\mathcal{W}_{3 \leftarrow 2}\left(U_{R}\right)$ and $U_{1}^{b}$ is below wave curve $\mathcal{W}_{3 \leftarrow 2}\left(U_{R}\right)$. This means that

$$
\phi_{3 \leftarrow 2}\left(U_{R}, U_{1}\right) \cdot \phi_{3 \leftarrow 2}\left(U_{R}, U_{1}^{b}\right)<0,
$$

where

$$
\begin{aligned}
& U_{1}=\left(h_{1}, u_{1}, \theta_{L}, a_{L}\right) \in \mathcal{W}_{1}\left(U_{L}\right), \\
& U_{2}=U_{1}^{b}=\left(h_{1}^{b}, u_{1}^{b}, \theta_{L}, a_{R}\right) \quad \text { when } \phi_{3 \leftarrow 2}\left(U_{R}, U_{1}^{b}\right)=0, \\
& h_{1}^{b} \text { is the bigger root of } \varphi\left(U_{1}, a_{R}\right)=0 .
\end{aligned}
$$

Third, we can determine $U_{3} \in \mathcal{W}_{2}\left(U_{2}\right)$, so $U_{3}=\left(\sqrt{h_{2}^{2} \theta_{L} / \theta_{R}}, u_{2}, \theta_{R}, a_{R}\right)$. Consequently, the Riemann problem for (1.1) has a solution of the form

$$
\mathcal{W}_{1}\left(U_{L}, U_{1}\right) \rightarrow \mathcal{W}_{4}\left(U_{1}, U_{2}\right) \rightarrow \mathcal{W}_{2}\left(U_{2}, U_{3}\right) \rightarrow \mathcal{W}_{3}\left(U_{3}, U_{R}\right)
$$

The construction (3.6) makes sense if $u_{2} \geq 0$, see Figure 3.5.

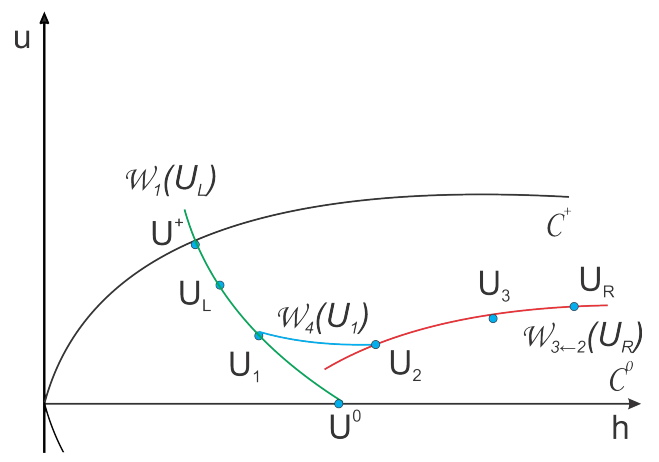

Figure 3.5: A Riemman solution of the form (3.6) in Construction B2.

Construction B3: A resonant case. Subcritical left-hand state and right-hand state near critical curve. Again, the resonant phenomenon may occur for these data, where the exact solution contains three waves which propagate with the same zero speed. Indeed, the solution first begins with a 1-rarefaction wave from $U_{L}$ to $U_{1}=U^{+}$, where

$$
U^{+}=\left(h^{+}, u^{+}, \theta_{L}, a_{L}\right)=\mathcal{R}_{1}\left(U_{L}\right) \cap \mathcal{C}^{+} .
$$

Second, for each bottom high level $a_{2} \in\left[a_{L}, a_{R}\right]$, the solution begins with a stationary wave from $U_{1}$ to $U_{2}$ using the smaller root of $\varphi\left(U_{1}, a_{2}\right)=0$ to shift $a_{1}$ to $a_{2}$, where

$$
U_{2}=\left(h_{2}, u_{2}, \theta_{L}, a_{2}\right) \in \mathcal{W}_{4}\left(U_{1}\right), \quad h_{2} \text { is a smaller root of } \varphi\left(U_{1}, a_{2}\right)=0 .
$$

Third, the next part of the solution is a zero-speed 1-shock from $U_{2}$ to $U_{3}=U_{2}^{\#}$, where $U_{2}^{\#}=\left(h_{2}^{\#}, u_{2}^{\#}, \theta_{L}, a_{2}\right)$ and $h_{2}^{\#}, u_{2}^{\#}$ are defined as in Lemma 2.1 
Fourth, the next part of the solution is a stationary wave from $U_{3}$ to $U_{4}$ to shift $a_{3}$ to $a_{R}$, where

$$
\left\{U_{4}\right\}=\mathcal{W}_{4 \rightarrow 1 \rightarrow 4}\left(U_{1}\right) \cap \mathcal{W}_{3 \leftarrow 2}\left(U_{R}\right) .
$$

We solve equation $\phi_{3 \leftarrow 2}\left(U_{R}, U_{4}\right)=0$ to find $a_{2}, U_{2}, U_{3}, U_{4}$, which admits a solution when $U_{3}^{b}$ is below wave curve $\mathcal{W}_{3 \leftarrow 2}\left(U_{R}\right)$ and $U_{3}$ is above wave curve $\mathcal{W}_{3 \leftarrow 2}\left(U_{R}\right)$. That is,

$$
\phi_{3 \leftarrow 2}\left(U_{R}, U_{3}^{b}\right) \cdot \phi_{3 \leftarrow 2}\left(U_{R}, U_{3}\right)<0,
$$

where

$$
\begin{aligned}
& U_{2}=\left(h_{2}, u_{2}, \theta_{L}, a_{2}\right) \in \mathcal{W}_{4}\left(U_{1}\right), \quad U_{3}=U_{2}^{\#}=\left(h_{2}^{\#}, u_{2}^{\#}, \theta_{L}, a_{2}\right), \\
& U_{4}=U_{3}^{b}=\left(h_{3}^{b}, u_{3}^{b}, \theta_{L}, a_{R}\right) \quad \text { when } \phi_{3 \leftarrow 2}\left(U_{R}, U_{3}^{b}\right)=0, \\
& h_{3}^{b} \text { is the bigger root of } \varphi\left(U_{3}, a_{R}\right)=0 .
\end{aligned}
$$

Fifth, we can determine $U_{5} \in \mathcal{W}_{2}\left(U_{4}\right)$, so $U_{5}=\left(\sqrt{h_{4}^{2} \theta_{L} / \theta_{R}}, u_{4}, \theta_{R}, a_{R}\right)$. Consequently, the Riemann problem for 1.1 has a solution of the form

$$
\mathcal{W}_{1}\left(U_{L}, U_{1}\right) \rightarrow \mathcal{W}_{4}\left(U_{1}, U_{2}\right) \rightarrow \mathcal{W}_{1}\left(U_{2}, U_{3}\right) \rightarrow \mathcal{W}_{4}\left(U_{3}, U_{4}\right) \rightarrow \mathcal{W}_{2}\left(U_{4}, U_{5}\right) \rightarrow \mathcal{W}_{3}\left(U_{5}, U_{R}\right)
$$

See Figure 3.6.



Figure 3.6: A Riemman solution of the form (3.7) in Construction B3.

\section{A Godunov-type scheme}

Relying on the constructions of Riemann solutions in the previous section, we are now in a position to build up a Godunov-type scheme for the system (1.1). Let us set

$$
U=\left(\begin{array}{c}
h \\
h u \\
h \theta \\
a
\end{array}\right), \quad F(U)=\left(\begin{array}{c}
h u \\
h u^{2}+\frac{g h^{2} \theta}{2} \\
h u \theta \\
0
\end{array}\right), \quad H(U)=\left(\begin{array}{c}
0 \\
-g h \theta \\
0 \\
0
\end{array}\right) .
$$


Then, the system $1.1-1.2$ can be written in form

$$
\partial_{t} U+\partial_{x} F(U)=H(U) \partial_{x} a
$$

Accordingly, given the initial condition

$$
U(x, 0)=U_{0}(x), \quad x \in \mathbb{R},
$$

then, the discrete initial values $U^{0}=\left(U_{j}^{0}\right)_{j \in \mathbb{Z}}$ are given by

$$
U_{j}^{0}=\frac{1}{\Delta x} \int_{x_{j-1 / 2}}^{x_{j+1 / 2}} U_{0}(x) d x, \quad j \in \mathbb{Z} .
$$

Suppose that $U^{n}=\left(U_{j}^{n}\right)_{j \in \mathbb{Z}}$ is known. We define the approximation $U^{n+1}=\left(U_{j}^{n+1}\right)_{j \in \mathbb{Z}}$ of $U\left(\cdot, t_{n+1}\right)$ as follows:

(i) We extend the sequence $U^{n}$ as a piecewise constant function $U_{\text {p.con }}\left(\cdot, t_{n}\right)$ defined by

$$
U_{\text {p.con }}\left(x, t_{n}\right)=U_{j}^{n}, \quad x_{j-1 / 2}<x<x_{j+1 / 2}, \quad j \in \mathbb{Z} .
$$

(ii) We solve the local Riemann problems for 4.1 with the initial condition

$$
U(x, 0)=U_{\text {p.con }}\left(x, t_{n}\right)
$$

to find the solution $U(\cdot, \Delta t)$. This solution is obtained by solving a juxtaposition of local Riemann problems, so

$$
U(x, t)=U_{\text {exact }}\left(\frac{x-x_{j+1 / 2}}{t} ; U_{j}^{n}, U_{j+1}^{n}\right), \quad x_{j}<x<x_{j+1}, \quad j \in \mathbb{Z},
$$

where $U_{\text {exact }}\left(x / t ; U_{L}, U_{R}\right)$ denote the exact solution of the Riemann problem for (4.1) corresponding to the Riemann data $\left(U_{L}, U_{R}\right)$.

(iii) We project $\left(\mathbb{L}^{2}\right.$-projection) the exact solution $U(\cdot, \Delta t)$ onto the piecewise constant functions, i.e.,

$$
U_{j}^{n+1}=\frac{1}{\Delta x} \int_{x_{j-1 / 2}}^{x_{j+1 / 2}} U(x, \Delta t) d x .
$$

Provided we assume the C.F.L. condition

$$
\frac{\Delta t}{\Delta x} \max \left\{\left|\lambda_{k}\left(U_{j}^{n}\right)\right|: k=1,2,3,4\right\} \leq \frac{1}{2},
$$

so that the waves issued from the points $x_{j-1 / 2}$ and $x_{j+1 / 2}$ do not interact. Since the $a$-component is constant in each interval $\left(x_{j-1 / 2}, x_{j+1 / 2}\right)$, then the right-hand side of (4.1) vanishes. The Godunov-type scheme is defined by

$$
U_{j}^{n+1}=U_{j}^{n}-\frac{\Delta t}{\Delta x}\left(F\left(U_{\text {exact }}\left(0-; U_{j}^{n}, U_{j+1}^{n}\right)\right)-F\left(U_{\text {exact }}\left(0+; U_{j-1}^{n}, U_{j}^{n}\right)\right)\right) .
$$


To complete the Godunov-type scheme (4.2), we will specify the values

$$
U_{\text {left }}:=U_{\text {exact }}\left(0-; U_{L}, U_{R}\right), \quad U_{\text {right }}:=U_{\text {exact }}\left(0+; U_{L}, U_{R}\right)
$$

as follows:

\begin{tabular}{c|c|c} 
Construction & $U_{\text {left }}$ & $U_{\text {right }}$ \\
\hline A1 3.2 & $U_{L}$ & $U_{1}$ \\
\hline A2 3.3 & $U_{1}$ & $U_{2}$ \\
\hline A3 3.4 & $U_{L}$ & $U_{3}$ \\
\hline B1 3.5 & $U_{1}$ & $U_{2}$ \\
\hline B2 3.6 & $U_{1}$ & $U_{2}$ \\
\hline B3 3.7 & $U_{1}$ & $U_{4}$
\end{tabular}

Riemann solver (A1). The Riemann solver (A1) relying on Construction A1 yields

$$
U_{\text {exact }}\left(0-; U_{L}, U_{R}\right)=U_{L}, \quad U_{\text {exact }}\left(0+; U_{L}, U_{R}\right)=U_{1}\left(U_{L}, U_{R}\right),
$$

where $U_{1}\left(U_{L}, U_{R}\right)=U_{L}^{s}=\left(h_{L}^{s}, u_{L}^{s}, \theta_{L}, a_{R}\right) \in \mathcal{W}_{4}\left(U_{L}\right), h_{L}^{s}$ is the smaller root of the nonlinear 2.12, $u_{L}^{s}=u_{L} h_{L} / h_{L}^{s}$. This implies that the Godunov scheme 4.2 using the Riemann solver (A1) becomes

$$
U_{j}^{n+1}=U_{j}^{n}-\frac{\Delta t}{\Delta x}\left(F\left(U_{j}^{n}\right)-F\left(U_{1}\left(U_{j-1}^{n}, U_{j}^{n}\right)\right)\right),
$$

where $U_{1}\left(U_{j-1}^{n}, U_{j}^{n}\right)$ is defined as in 4.3.

Riemann solver (A2). The Riemann solver (A2) relying on Construction A2 yields

$$
U_{\text {exact }}\left(0-; U_{L}, U_{R}\right)=U_{1}\left(U_{L}, U_{R}\right), \quad U_{\text {exact }}\left(0+; U_{L}, U_{R}\right)=U_{2}\left(U_{L}, U_{R}\right) .
$$

We can determine $U_{1}\left(U_{L}, U_{R}\right)$ and $U_{2}\left(U_{L}, U_{R}\right)$ as follows:

Step 1.

- Set $h_{1}=h_{L}^{\#}$, where $h_{L}^{\#}$ is defined as in Lemma 2.1.

- Set $h_{2}=h^{0}$, where $U^{0}=\left(h^{0}, u^{0}, \theta_{L}, a_{L}\right)=\mathcal{W}_{1}\left(U_{L}\right) \cap \mathcal{C}^{0}$.

Step 2.

- Estimate $h_{T}=\left(h_{1}+h_{2}\right) / 2$.

- Compute $U_{T}=\left(h_{T}, u_{T}, \theta_{L}, a_{L}\right) \in \mathcal{W}_{1}\left(U_{L}\right)$. 
- Compute $U_{T}^{b}=\left(h_{T}^{b}, u_{T}^{b}, \theta_{L}, a_{R}\right) \in \mathcal{W}_{4}\left(U_{T}\right)$, where $h_{T}^{b}$ is the bigger root of the nonlinear equation 2.12), $u_{T}^{b}=u_{T} h_{T} / h_{T}^{b}$.

Step 3.

- If $U_{T}^{b} \in \mathcal{W}_{3 \leftarrow 2}\left(U_{R}\right)$, terminate the computation and set $U_{1}\left(U_{L}, U_{R}\right)=U_{T}, U_{2}\left(U_{L}\right.$, $\left.U_{R}\right)=U_{T}^{b}$.

- If $U_{T}^{b}$ is above the curve $\mathcal{W}_{3 \leftarrow 2}\left(U_{R}\right)$, set $h_{1}=h$ and return Step 2.

- If $U_{T}^{b}$ is below the curve $\mathcal{W}_{3 \leftarrow 2}\left(U_{R}\right)$, set $h_{2}=h$ and return Step 2 .

This implies that the Godunov scheme 4.2) using the Riemann solver (A2) becomes

$$
U_{j}^{n+1}=U_{j}^{n}-\frac{\Delta t}{\Delta x}\left(F\left(U_{1}\left(U_{j}^{n}, U_{j+1}^{n}\right)\right)-F\left(U_{2}\left(U_{j-1}^{n}, U_{j}^{n}\right)\right)\right)
$$

where $U_{1}\left(U_{j}^{n}, U_{j+1}^{n}\right), U_{2}\left(U_{j-1}^{n}, U_{j}^{n}\right)$ are defined as in 4.4).

Riemann solver (A3). The Riemann solver (A3) relying on Construction A3 yields

$$
U_{\text {exact }}\left(0-; U_{L}, U_{R}\right)=U_{L}, \quad U_{\text {exact }}\left(0+; U_{L}, U_{R}\right)=U_{3}\left(U_{L}, U_{R}\right)
$$

We can determine $U_{3}\left(U_{L}, U_{R}\right)$ as follows:

Step 1. Set $a_{1}=a_{L}, a_{2}=a_{R}$.

Step 2.

- Estimate $a=\left(a_{1}+a_{2}\right) / 2$.

- Compute $U_{L}^{s}=\left(h_{L}^{s}, u_{L}^{s}, \theta_{L}, a\right)$ where $h_{L}^{s}$ is the smaller root of the nonlinear equation (2.12), $u_{L}^{s}=u_{L} h_{L} / h_{L}^{s}$.

- Compute $U_{L}^{s \#}=\left(h_{L}^{s \#}, u_{L}^{s \#}, \theta_{L}, a\right)$ where $h_{L}^{s \#}, u_{L}^{s \#}$ are defined as in Lemma 2.1.

- Compute $U_{L}^{s \# b}=\left(h_{L}^{s \# b}, u_{L}^{s \# b}, \theta_{L}, a_{R}\right)$ where $h_{L}^{s \# b}$ is the bigger root of the nonlinear equation (2.12), $u_{L}^{s \# b}=u_{L}^{s \#} h_{L}^{s \#} / h_{L}^{s \# b}$.

Step 3.

- If $U_{L}^{s \# b} \in \mathcal{W}_{3 \leftarrow 2}\left(U_{R}\right)$, terminate the computation and set $U_{3}\left(U_{L}, U_{R}\right)=U_{L}^{s \# b}$.

- If $U_{L}^{s \# b}$ is above the curve $\mathcal{W}_{3 \leftarrow 2}\left(U_{R}\right)$, set $a_{2}=a$ and return Step 2 .

- If $U_{L}^{s \# b}$ is below the curve $\mathcal{W}_{3 \leftarrow 2}\left(U_{R}\right)$, set $a_{1}=a$ and return Step 2 . 
This implies that the Godunov scheme 4.2 using the Riemann solver (A3) becomes

$$
U_{j}^{n+1}=U_{j}^{n}-\frac{\Delta t}{\Delta x}\left(F\left(U_{j}^{n}\right)-F\left(U_{3}\left(U_{j-1}^{n}, U_{j}^{n}\right)\right)\right)
$$

where $U_{3}\left(U_{j-1}^{n}, U_{j}^{n}\right)$ is defined as in 4.5.

Riemann solver (B1). The Riemann solver (B1) relying on Construction B1 yields

$$
U_{\text {exact }}\left(0-; U_{L}, U_{R}\right)=U_{1}\left(U_{L}, U_{R}\right), \quad U_{\text {exact }}\left(0+; U_{L}, U_{R}\right)=U_{2}\left(U_{L}, U_{R}\right),
$$

where

$$
\begin{aligned}
& U_{1}\left(U_{L}, U_{R}\right)=U^{+}=\left(h^{+}, u^{+}, \theta_{L}, a_{L}\right)=\mathcal{W}_{1}\left(U_{L}\right) \cap \mathcal{C}^{+}, \\
& U_{2}\left(U_{L}, U_{R}\right)=U^{+s}=\left(h^{+s}, u^{+s}, \theta_{L}, a_{R}\right),
\end{aligned}
$$

$h^{+s}$ is the smaller root of the nonlinear equation (2.12) and $u^{+s}=u^{+} h^{+} / h^{+s}$. This implies that the Godunov scheme 4.2 using the Riemann solver (B1) becomes

$$
U_{j}^{n+1}=U_{j}^{n}-\frac{\Delta t}{\Delta x}\left(F\left(U_{1}\left(U_{j}^{n}, U_{j+1}^{n}\right)\right)-F\left(U_{3}\left(U_{j-1}^{n}, U_{j}^{n}\right)\right)\right),
$$

where $U_{1}\left(U_{j}^{n}, U_{j+1}^{n}\right), U_{3}\left(U_{j-1}^{n}, U_{j}^{n}\right)$ are defined as in 4.6 .

Riemann solver (B2). The Riemann solver (B2) relying on Construction B2 yields

$$
U_{\text {exact }}\left(0-; U_{L}, U_{R}\right)=U_{1}\left(U_{L}, U_{R}\right), \quad U_{\text {exact }}\left(0+; U_{L}, U_{R}\right)=U_{2}\left(U_{L}, U_{R}\right)
$$

We can determine $U_{1}\left(U_{L}, U_{R}\right)$ and $U_{2}\left(U_{L}, U_{R}\right)$ as follows:

Step 1.

- Set $h_{1}=h^{+}$, where $U^{+}=\left(h^{+}, u^{+}, \theta_{L}, a_{L}\right)=\mathcal{W}_{1}\left(U_{L}\right) \cap \mathcal{C}^{+}$.

- Set $h_{2}=h^{0}$, where $U^{0}=\left(h^{0}, u^{0}, \theta_{L}, a_{L}\right)=\mathcal{W}_{1}\left(U_{L}\right) \cap \mathcal{C}^{0}$.

Step 2.

- Estimate $h_{T}=\left(h_{1}+h_{2}\right) / 2$.

- Compute $U_{T}=\left(h_{T}, u_{T}, \theta_{L}, a_{L}\right) \in \mathcal{W}_{1}\left(U_{L}\right)$.

- Compute $U_{T}^{b}=\left(h_{T}^{b}, u_{T}^{b}, \theta_{L}, a_{R}\right) \in \mathcal{W}_{4}\left(U_{T}\right)$, where $h_{T}^{b}$ is the bigger root of the nonlinear equation 2.12, $u_{T}^{b}=u_{T} h_{T} / h_{T}^{b}$.

Step 3.

- If $U_{T}^{b} \in \mathcal{W}_{3 \leftarrow 2}\left(U_{R}\right)$, terminate the computation and set $U_{1}\left(U_{L}, U_{R}\right)=U_{T}, U_{2}\left(U_{L}\right.$, $\left.U_{R}\right)=U_{T}^{b}$. 
- If $U_{T}^{b}$ is above the curve $\mathcal{W}_{3 \leftarrow 2}\left(U_{R}\right)$, set $h_{1}=h$ and return Step 2 .

- If $U_{T}^{b}$ is below the curve $\mathcal{W}_{3 \leftarrow 2}\left(U_{R}\right)$, set $h_{2}=h$ and return Step 2.

This implies that the Godunov scheme (4.2) using the Riemann solver (B2) becomes

$$
U_{j}^{n+1}=U_{j}^{n}-\frac{\Delta t}{\Delta x}\left(F\left(U_{1}\left(U_{j}^{n}, U_{j+1}^{n}\right)\right)-F\left(U_{2}\left(U_{j-1}^{n}, U_{j}^{n}\right)\right)\right),
$$

where $U_{1}\left(U_{j}^{n}, U_{j+1}^{n}\right), U_{2}\left(U_{j-1}^{n}, U_{j}^{n}\right)$ are defined as in 4.7).

Riemann solver (B3). The Riemann solver (B3) relying on Construction B3 yields

$$
U_{\text {exact }}\left(0-; U_{L}, U_{R}\right)=U_{1}\left(U_{L}, U_{R}\right), \quad U_{\text {exact }}\left(0+; U_{L}, U_{R}\right)=U_{4}\left(U_{L}, U_{R}\right) .
$$

We can determine $U_{1}\left(U_{L}, U_{R}\right)$ and $U_{4}\left(U_{L}, U_{R}\right)$ as follows:

Step 1. $U_{1}\left(U_{L}, U_{R}\right)=U^{+}=\left(h^{+}, u^{+}, \theta_{L}, a_{L}\right)=\mathcal{W}_{1}\left(U_{L}\right) \cap \mathcal{C}^{+}$.

Step 2. Set $a_{1}=a_{L}, a_{2}=a_{R}$.

Step 3.

- Estimate $a=\left(a_{1}+a_{2}\right) / 2$.

- Compute $U^{+s}=\left(h^{+s}, u^{+s}, \theta_{L}, a\right)$ where $h^{+s}$ is the smaller root of the nonlinear equation 2.12, $u^{+s}=u^{+} h^{+} / h^{+s}$.

- Compute $U^{+s \#}=\left(h^{+s \#}, u^{+s \#}, \theta_{L}, a\right)$ where $h^{+s \#}, u^{+s \#}$ are defined as in Lemma 2.1 .

- Compute $U^{+s \# b}=\left(h^{+s \# b}, u^{+s \# b}, \theta_{L}, a_{R}\right)$ where $h^{+s \# b}$ is the bigger root of the nonlinear equation 2.12$), u^{+s \# b}=u^{+s \#} h^{+s \#} / h^{+s \# b}$.

Step 4 .

- If $U^{+s \# b} \in \mathcal{W}_{3 \leftarrow 2}\left(U_{R}\right)$, terminate the computation and set $U_{4}\left(U_{L}, U_{R}\right)=U^{+s \# b}$.

- If $U^{+s \# b}$ is above the curve $\mathcal{W}_{3 \leftarrow 2}\left(U_{R}\right)$, set $a_{2}=a$ and return Step 3 .

- If $U^{+s \# b}$ is below the curve $\mathcal{W}_{3 \leftarrow 2}\left(U_{R}\right)$, set $a_{1}=a$ and return Step 3 .

This implies that the Godunov scheme (4.2) using the Riemann solver (B3) becomes

$$
U_{j}^{n+1}=U_{j}^{n}-\frac{\Delta t}{\Delta x}\left(F\left(U_{1}\left(U_{j}^{n}, U_{j+1}^{n}\right)\right)-F\left(U_{4}\left(U_{j-1}^{n}, U_{j}^{n}\right)\right)\right),
$$

where $U_{1}\left(U_{j}^{n}, U_{j+1}^{n}\right), U_{4}\left(U_{j-1}^{n}, U_{j}^{n}\right)$ are defined as in 4.8).

Theorem 4.1 (Properties). The Godunov-type scheme (4.2) possesses the following properties: 
(i) It is a well-balanced scheme, that is, it is capable of capturing stationary waves exactly.

(ii) It preserves the positivity of the water height.

Proof. (i) Suppose that $U^{n}$ is a stationary wave, then on each cell $x_{j-1 / 2}<x<x_{j+1 / 2}$, $t^{n}<t \leq t^{n+1}$, the exact Riemann solution is constant, $j \in \mathbb{Z}, n=0,1,2, \ldots$. This implies that

$$
\begin{aligned}
& U_{\text {exact }}\left(\frac{x-x_{j+1 / 2}}{t} ; U_{j}^{n}, U_{j+1}^{n}\right)= \begin{cases}U_{j}^{n} & \text { if } x<x_{j+1 / 2}, \\
U_{j+1}^{n} & \text { if } x>x_{j+1 / 2},\end{cases} \\
& U_{\text {exact }}\left(\frac{x-x_{j-1 / 2}}{t} ; U_{j-1}^{n}, U_{j}^{n}\right)= \begin{cases}U_{j-1}^{n} & \text { if } x<x_{j-1 / 2}, \\
U_{j}^{n} & \text { if } x>x_{j-1 / 2},\end{cases}
\end{aligned}
$$

SO

$$
U_{\text {exact }}\left(0-; U_{j}^{n}, U_{j+1}^{n}\right)=U_{\text {exact }}\left(0+; U_{j-1}^{n}, U_{j}^{n}\right)
$$

which yields

$$
U_{j}^{n+1}=U_{j}^{n}, \quad \forall j \in \mathbb{Z}, n=0,1,2, \cdots
$$

So, if the initial data $U^{0}$ corresponds to a stationary wave, then it also holds that

$$
U_{j}^{n}=U_{j}^{0}, \quad \forall j \in \mathbb{Z}, n=0,1,2, \ldots
$$

This establishes (i).

(ii) To prove this, we need to find $U_{\text {exact }}\left(0-; U_{j}^{n}, U_{j+1}^{n}\right), U_{\text {exact }}\left(0+; U_{j-1}^{n}, U_{j}^{n}\right)$ in the Godunov-type scheme through on 6 constructions in Section 3 , so we have 36 pairs of constructions as $\{\mathrm{A} 1-\mathrm{A} 1 ; \mathrm{A} 1-\mathrm{A} 2 ; \ldots\}$ to determine exact solutions. In each pair, we show that high of water and temperature water always are positive. We need only to choose a pair to prove this, since the other cases are similar.

Let us choose Case B2-A3 and suppose that $h_{j}^{n}>0, u_{j}^{n}>0, \theta_{j}^{n}>0(\forall j \in \mathbb{Z})$, we have

$$
\begin{aligned}
U_{j}^{n+1} & =U_{j}^{n}-\frac{\Delta t}{\Delta x}\left(F\left(U_{\text {exact }}\left(0-; U_{j}^{n}, U_{j+1}^{n}\right)\right)-F\left(U_{\text {exact }}\left(0+; U_{j-1}^{n}, U_{j}^{n}\right)\right)\right) \\
& =U_{j}^{n}-\frac{\Delta t}{\Delta x}\left(F\left(U_{1}\left(U_{j}^{n}, U_{j+1}^{n}\right)\right)-F\left(U_{3}\left(U_{j-1}^{n}, U_{j}^{n}\right)\right)\right),
\end{aligned}
$$

where $U_{1}\left(U_{j}^{n}, U_{j+1}^{n}\right)=\left(U_{j}^{n}\right)^{\mathcal{W}_{1}} \in \mathcal{W}_{1}\left(U_{j}^{n}\right)$ and $U_{3}\left(U_{j-1}^{n}, U_{j}^{n}\right)=\left(U_{j-1}\right)^{s \# b}$ are defined by Construction B2, Construction A3, respectively.

First, we point out that $h_{j}^{n+1}>0, \forall j \in \mathbb{Z}$. It holds from 4.9 that

$$
\begin{aligned}
h_{j}^{n+1} & =h_{j}^{n}-\frac{\Delta t}{\Delta x}\left(\left(h_{j}^{n}\right)^{\mathcal{W}_{1}}\left(u_{j}^{n}\right)^{\mathcal{W}_{1}}-\left(h_{j-1}^{n}\right)^{s \# b}\left(u_{j-1}^{n}\right)^{s \# b}\right) \\
& =h_{j}^{n}-\frac{\Delta t}{\Delta x}\left(h_{j}^{n}\right)^{\mathcal{W}_{1}}\left(u_{j}^{n}\right)^{\mathcal{W}_{1}}+\frac{\Delta t}{\Delta x}\left(h_{j-1}^{n}\right)^{s \# b}\left(u_{j-1}^{n}\right)^{s \# b} .
\end{aligned}
$$


If $\left(U_{j}^{n}\right)^{\mathcal{W}_{1}}=\left(U_{j}^{n}\right)^{\text {rare }} \in \mathcal{R}_{1}\left(U_{j}^{n}\right)$ then $\left(h_{j}^{n}\right)^{\text {rare }}<h_{j}^{n}$, we set $\left(h_{j}^{n}\right)^{\text {rare }}=h_{j}^{n} \cdot q,(0<q<1)$, we have

$$
h_{j}^{n+1}=h_{j}^{n}\left(1-\frac{\Delta t}{\Delta x} \cdot q \cdot\left(u_{j}^{n}\right)^{\text {rare }}\right)+\frac{\Delta t}{\Delta x}\left(\left(h_{j-1}^{n}\right)^{s \# b}\left(u_{j-1}^{n}\right)^{s \# b}\right) .
$$

Next, the C.F.L. condition

$$
\frac{\Delta t}{\Delta x} \max \left\{\left|\lambda_{k}\left(U_{j}^{n}\right)^{\mathrm{rare}}\right|: k=1,2,3,4\right\} \leq \frac{1}{2}
$$

implies that

$$
1-\frac{\Delta t}{\Delta x} \cdot q \cdot\left(u_{j}^{n}\right)^{\mathrm{rare}}>0
$$

If $\left(U_{j}^{n}\right)^{\mathcal{W}_{1}}=\left(U_{j}^{n}\right)^{\text {shock }} \in \mathcal{S}_{1}\left(U_{j}^{n}\right)$ then $\left(h_{j}^{n}\right)^{\text {shock }}>h_{j}^{n}$, applying the Rankine-Hugoniot relations, we have

$$
\begin{aligned}
h_{j}^{n+1}= & h_{j}^{n}-\frac{\Delta t}{\Delta x}\left(h_{j}^{n}\right)^{\text {shock }}\left(u_{j}^{n}\right)^{\text {shock }}+\frac{\Delta t}{\Delta x}\left(\left(h_{j-1}^{n}\right)^{s \# b}\left(u_{j-1}^{n}\right)^{s \# b}\right) \\
= & h_{j}^{n}\left(1-\frac{\Delta t}{\Delta x}\left(u_{j}^{n}\right)\right)-\frac{\Delta t}{\Delta x} \sigma\left(U_{j}^{n},\left(U_{j}^{n}\right)^{\text {shock }}\right)\left(\left(h_{j}^{n}\right)^{\text {shock }}-h_{j}^{n}\right) \\
& +\frac{\Delta t}{\Delta x}\left(\left(h_{j-1}^{n}\right)^{s \# b}\left(u_{j-1}^{n}\right)^{s \# b}\right)
\end{aligned}
$$

where

so that

$$
\frac{\Delta t}{\Delta x} \max \left\{\left|\lambda_{k}\left(U_{j}^{n}\right)\right|: k=1,2,3,4\right\} \leq \frac{1}{2}, \quad \sigma\left(U_{j}^{n},\left(U_{j}^{n}\right)^{\text {shock }}\right)<0,
$$

$$
h_{j}^{n}\left(1-\frac{\Delta t}{\Delta x}\left(u_{j}^{n}\right)\right)-\frac{\Delta t}{\Delta x} \sigma\left(U_{j}^{n},\left(U_{j}^{n}\right)^{\text {shock }}\right)\left(\left(h_{j}^{n}\right)^{\text {shock }}-h_{j}^{n}\right)>0 .
$$

Besides, it holds that

$$
\begin{aligned}
\left(h_{j-1}^{n}\right)^{s \# b}\left(u_{j-1}^{n}\right)^{s \# b} & =\left(h_{j-1}^{n}\right)^{s \#}\left(u_{j-1}^{n}\right)^{s \#} & & \text { (Stationary wave) } \\
& =\left(h_{j-1}^{n}\right)^{s}\left(u_{j-1}^{n}\right)^{s} & & \text { (Lemma 2.1) } \\
& =h_{j-1}^{n} u_{j-1}^{n}>0 . & & \text { (Stationary wave) }
\end{aligned}
$$

From 4.10, 4.11, 4.12, 4.13, we conclude that $h_{j}^{n+1}>0$.

Second, we prove $\theta_{j}^{n+1}>0, \forall j \in \mathbb{Z}$, beginning with $h \theta$ component in scheme 4.9):

$$
\begin{aligned}
h_{j}^{n+1} \theta_{j}^{n+1} & =h_{j}^{n} \theta_{j}^{n}-\frac{\Delta t}{\Delta x}\left(\left(h_{j}^{n}\right)^{\mathcal{W}_{1}}\left(u_{j}^{n}\right)^{\mathcal{W}_{1}}\left(\theta_{j}^{n}\right)^{\mathcal{W}_{1}}-\left(h_{j-1}^{n}\right)^{s \# b}\left(u_{j-1}^{n}\right)^{s \# b}\left(\theta_{j-1}^{n}\right)^{s \# b}\right) \\
& =h_{j}^{n} \theta_{j}^{n}-\frac{\Delta t}{\Delta x}\left(\left(h_{j}^{n}\right)^{\mathcal{W}_{1}}\left(u_{j}^{n}\right)^{\mathcal{W}_{1}} \theta_{j}^{n}-\left(h_{j-1}^{n}\right)^{s \# b}\left(u_{j-1}^{n}\right)^{s \# b} \theta_{j-1}^{n}\right) \\
& =\theta_{j}^{n}\left(h_{j}^{n}-\frac{\Delta t}{\Delta x}\left(h_{j}^{n}\right)^{\mathcal{W}_{1}}\left(u_{j}^{n}\right)^{\mathcal{W}_{1}}\right)+\theta_{j-1}^{n} \frac{\Delta t}{\Delta x}\left(h_{j-1}^{n}\right)^{s \# b}\left(u_{j-1}^{n}\right)^{s \# b} .
\end{aligned}
$$

Arguing similarly as above, we obtain

$$
h_{j}^{n}-\frac{\Delta t}{\Delta x}\left(h_{j}^{n}\right)^{\mathcal{W}_{1}}\left(u_{j}^{n}\right)^{\mathcal{W}_{1}}>0, \quad \frac{\Delta t}{\Delta x}\left(h_{j-1}^{n}\right)^{s \# b}\left(u_{j-1}^{n}\right)^{s \# b}>0 .
$$

From 4.14 and 4.15, we conclude that $\theta_{j}^{n+1}>0$. This establishes (ii). 


\section{Numerical experiments}

This section is devoted to numerical tests by using MATLAB, which demonstrate the advantages of our scheme (4.2). For each test, we compare the approximate solution $U_{h}$ corresponding to the mesh size $h$ with the exact solution $U$ by computing the accuracy in the $L^{1}$-norm. By using the stability condition $\mathrm{CFL}=0.5$, and we plot the solution $U_{h}$ and $U$ for $x \in[-1,1], t=0.05$.

\subsection{Test 1: Well-balanced}

\subsubsection{Test 1.1: Well-balanced with discontinuous topography}

This test is aimed to demonstrate that the Godunov-type scheme 4.2 is capable of maintaining equilibrium states when topography is discontinuous. Let

$$
U_{0}(x)= \begin{cases}U_{L}=\left(h_{L}, u_{L}, \theta_{L}, a_{L}\right) & \text { if } x<0, \\ U_{R}=\left(h_{R}, u_{R}, \theta_{R}, a_{R}\right) & \text { if } x>0,\end{cases}
$$

where the left-hand and the right-hand states of a stationary wave. Here they are chosen to be by

$$
\left(h_{L}, u_{L}, \theta_{L}, a_{L}\right)=(3,0.5,3,1), \quad\left(h_{R}, u_{R}, \theta_{R}, a_{R}\right)=(2.799369,0.535835,3,1.2) .
$$
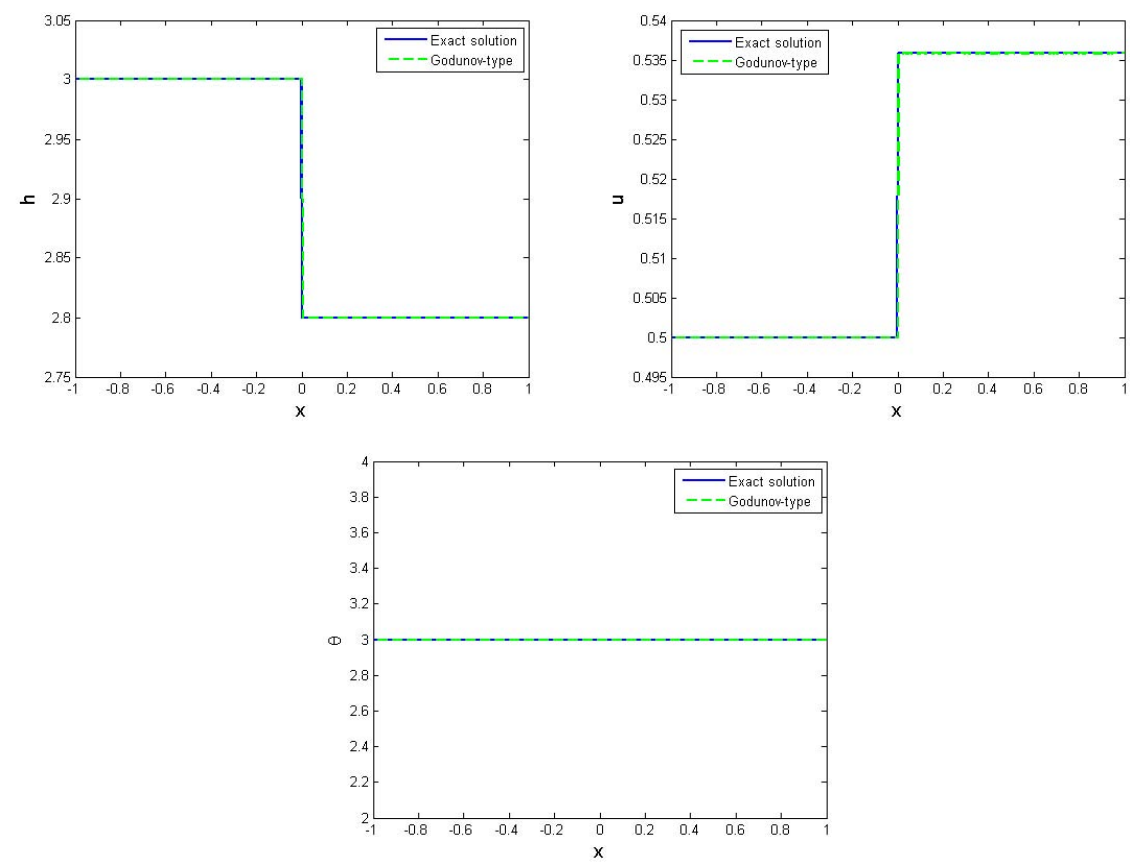

Figure 5.1: A stationary contact wave is captured exactly by Godunov method using our Riemann solver with 800 mesh points in Test 1.1. 
See Figure 5.1, and Table 5.1 shows that the stationary contact is well captured by Godunov method using our exact Riemann solver for $x \in[-1,1]$ with 800 mesh points and at time $t=0.05$.

\begin{tabular}{|c|c|}
\hline$N$ & $\left\|U_{h}-U\right\|_{L^{1}}$ \\
\hline 400 & 0.0015 \\
800 & $7.2967 \times 10^{-4}$ \\
\hline
\end{tabular}

Table 5.1: Errors of numerical approximations for different mesh sizes for Test 1.1.

\subsubsection{Test 1.2: Well-balanced with flat topography}

This test is aimed to demonstrate that the Godunov-type scheme 4.2 is capable of maintaining equilibrium states when topography is flat and velocity of water equals zero.
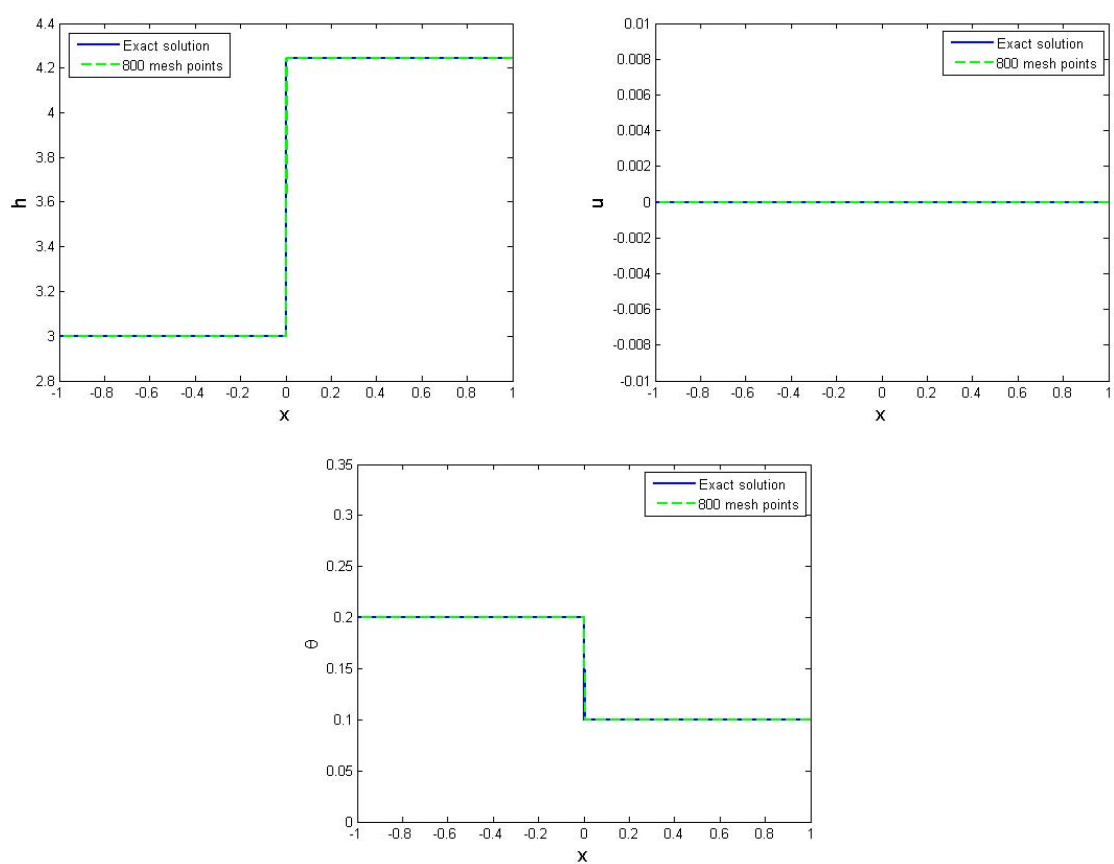

Figure 5.2: A stationary contact wave is captured exactly by Godunov method using our Riemann solver with 800 mesh points in Test 1.2.

Let

$$
U_{0}(x)= \begin{cases}U_{L}=\left(h_{L}, u_{L}, \theta_{L}, a_{L}\right) & \text { if } x<0, \\ U_{R}=\left(h_{R}, u_{R}, \theta_{R}, a_{R}\right) & \text { if } x>0\end{cases}
$$


where the left-hand and the right-hand states of a stationary wave. Here they are chosen to be by

$$
\left(h_{L}, u_{L}, \theta_{L}, a_{L}\right)=(3,0,0.2,2), \quad\left(h_{R}, u_{R}, \theta_{R}, a_{R}\right)=(4.2426,0,0.1,2) .
$$

See Figure 5.2, and Table 5.2 shows that the stationary contact is well captured by Godunov method using our exact Riemann solver for $x \in[-1,1]$ with 800 mesh points and at time $t=0.05$.

\begin{tabular}{|c|c|}
\hline$N$ & $\left\|U_{h}-U\right\|_{L^{1}}$ \\
\hline 400 & $2.8377 \times 10^{-17}$ \\
800 & $2.8066 \times 10^{-17}$ \\
\hline
\end{tabular}

Table 5.2: Errors of numerical approximations for different mesh sizes for Test 1.2.

\subsubsection{Test 1.3: Well-balanced with continuous topography}

This test is aimed to demonstrate that the Godunov-type scheme 4.2 is capable of maintaining equilibrium states when topography is continuous. Let us take the smooth topography as $a(x)=2-\tan ^{-1}(x)$ and the initial data as $\left(h_{0}, u_{0}, \theta_{0}, a_{0}\right)=(0.5,4,3,2)$.
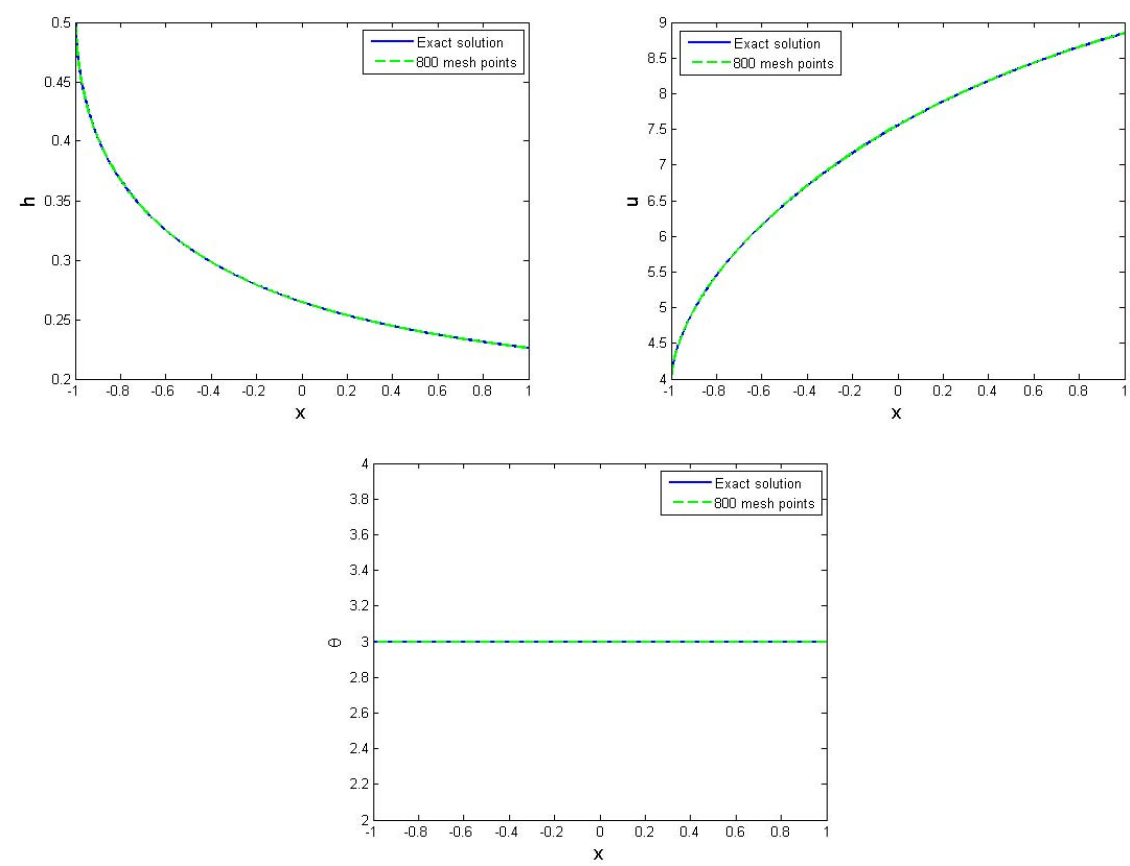

Figure 5.3: A stationary contact wave is captured exactly by Godunov method using our Riemann solver with 800 mesh points in Test 1.3. 
See Figure 5.3, and Table 5.3 shows that the stationary contact is well captured by Godunov method using our exact Riemann solver for $x \in[-1,1]$ with 800 mesh points and at time $t=0.05$.

\begin{tabular}{|c|c|}
\hline$N$ & $\left\|U_{h}-U\right\|_{L^{1}}$ \\
\hline 400 & $2.3946 \times 10^{-5}$ \\
800 & $6.4209 \times 10^{-5}$ \\
\hline
\end{tabular}

Table 5.3: Errors of numerical approximations for different mesh sizes for Test 1.3.

\subsection{Test 2: Construction A1}

In this test we approximate a Riemann solution of Construction A1. The Riemann data are given by

$$
U_{0}(x)= \begin{cases}U_{L}=\left(h_{L}, u_{L}, \theta_{L}, a_{L}\right)=(0.01,4,3,1.2) \in G_{1} & \text { if } x<0 \\ U_{R}=\left(h_{R}, u_{R}, \theta_{R}, a_{R}\right)=(0.02,2,4,1) \in G_{1} & \text { if } x>0 .\end{cases}
$$

According to Construction A1, the exact solution is a stationary wave from $U_{L}$ to $U_{1}$, followed by a 1-shock wave from $U_{1}$ to $U_{2}$, followed by a 2-contact wave from $U_{2}$ to $U_{3}$, then followed by a 3 -shock wave from $U_{3}$ to $U_{R}$, where $U_{L}, U_{1}, U_{2}, U_{3}, U_{R}$ are reported in Table 5.4 .

\begin{tabular}{c|ccccc}
\hline & $U_{L}$ & $U_{1}$ & $U_{2}$ & $U_{3}$ & $U_{R}$ \\
\hline$h$ & 0.01 & 0.007573 & 0.055304 & 0.047894 & 0.02 \\
$u$ & 4.0 & 5.282064 & 3.039743 & 3.039743 & 2.0 \\
$\theta$ & 3.0 & 3.0 & 3.0 & 4.0 & 4.0 \\
$a$ & 1.2 & 1.0 & 1.0 & 1.0 & 1.0 \\
\hline
\end{tabular}

Table 5.4: States that separate the elementary waves of the exact solution of the Riemann problem in Test 2: Construction A1.

The errors of solution and orders of accuracy for Test 2 are reported in Table 5.5 .

Figure 5.4 shows that the approximate solutions get closer to the exact solution when the mesh sizes get smaller. Notably, Table 5.5 shows that the errors become smaller and orders of accuracy are good as the mesh size gets smaller. Besides that, when the initial height of water is very small and nearly zero, our scheme still ensures that height is greater than zero. So, Test 2 demonstrates the convergence of the approximate solutions by Godunov-type scheme to the exact solution and the positive height of water. 


\begin{tabular}{|c|c|c|}
\hline$N$ & $\left\|U_{h}-U\right\|_{L^{1}}$ & Order \\
\hline 100 & 0.1127 & \\
200 & 0.0799 & 0.50 \\
400 & 0.0450 & 0.83 \\
800 & 0.0277 & 0.70 \\
1600 & 0.0170 & 0.70 \\
\hline
\end{tabular}

Table 5.5: Errors of numerical approximations and orders of accuracy for different mesh sizes for Test 2 .
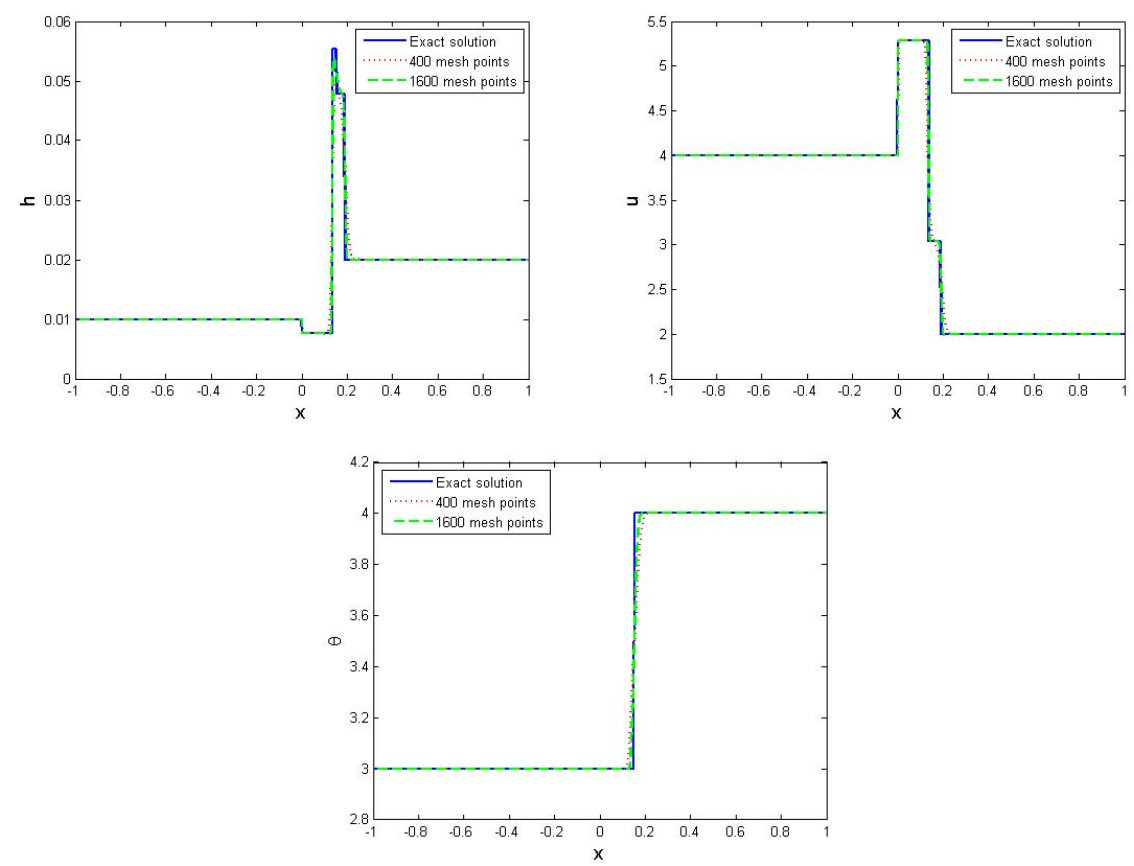

Figure 5.4: Exact solution and approximate solutions for different mesh sizes for Test 2.

\subsection{Test 3: Construction A2}

In this test we approximate a Riemann solution of Construction A2. We consider the Riemann data to be

$$
U_{0}(x)= \begin{cases}U_{L}=\left(h_{L}, u_{L}, \theta_{L}, a_{L}\right)=(0.5,4,3,1.5) \in G_{1} & \text { if } x<0, \\ U_{R}=\left(h_{R}, u_{R}, \theta_{R}, a_{R}\right)=(1.2,1.5,4,1) \in G_{2} & \text { if } x>0 .\end{cases}
$$

According to Construction A2, the exact solution is a 1-shock wave from $U_{L}$ to $U_{1}$, followed by a stationary wave from $U_{1}$ to $U_{2}$, followed by a 2-contact wave from $U_{2}$ to $U_{3}$, then 
followed by a 3-rarefaction wave from $U_{3}$ to $U_{R}$, where $U_{L}, U_{1}, U_{2}, U_{3}, U_{R}$ are reported in Table 5.6 ,

\begin{tabular}{c|ccccc}
\hline & $U_{L}$ & $U_{1}$ & $U_{2}$ & $U_{3}$ & $U_{R}$ \\
\hline$h$ & 0.5 & 0.780210 & 1.327153 & 1.149348 & 1.2 \\
$u$ & 4.0 & 2.053781 & 1.207381 & 1.207381 & 1.5 \\
$\theta$ & 3.0 & 3.0 & 3.0 & 4.0 & 4.0 \\
$a$ & 1.5 & 1.5 & 1.0 & 1.0 & 1.0 \\
\hline
\end{tabular}

Table 5.6: States that separate the elementary waves of the exact solution of the Riemann problem in Test 3: Construction A2.


Figure 5.5: Exact solution and approximate solutions for different mesh sizes for Test 3.

The errors of solution and orders of accuracy for Test 3 are reported in Table 5.7 .

Figure 5.5 shows that the approximate solutions get closer to the exact solution when the mesh sizes get smaller. Notably, Table 5.7 shows that the errors become smaller and orders of accuracy are good as the mesh size gets smaller. So, Test 3 demonstrates the convergence of the approximate solutions by Godunov-type scheme to the exact solution when the initial data belong to Supercritical and subcritical. 


\begin{tabular}{|c|c|c|}
\hline$N$ & $\left\|U_{h}-U\right\|_{L^{1}}$ & Order \\
\hline 100 & 0.1016 & \\
200 & 0.0672 & 0.60 \\
400 & 0.0371 & 0.86 \\
800 & 0.0232 & 0.68 \\
1600 & 0.0152 & 0.61 \\
\hline
\end{tabular}

Table 5.7: Errors of numerical approximations and orders of accuracy for different mesh sizes for Test 3 .

\subsection{Test 4: A resonant phenomenon case (Construction A3)}

In this test we approximate a Riemann solution of Construction A3, where the Riemann data are given by

$$
U_{0}(x)= \begin{cases}U_{L}=\left(h_{L}, u_{L}, \theta_{L}, a_{L}\right)=(0.5,4,3,1.5) \in G_{1} & \text { if } x<0 \\ U_{R}=\left(h_{R}, u_{R}, \theta_{R}, a_{R}\right)=(1,3.5,4,1) \in G_{2} & \text { if } x>0\end{cases}
$$

According to Construction A3, the exact solution is a stationary wave from $U_{L}$ to $U_{1}$, followed by a 1 -shock wave with zero-speed from $U_{1}$ to $U_{2}$, followed by a stationary wave from $U_{2}$ to $U_{3}$, followed by a 2-contact wave from $U_{3}$ to $U_{4}$, then followed by a 3-rarefaction wave from $U_{4}$ to $U_{R}$, where $U_{L}, U_{1}, U_{2}, U_{3}, U_{4}, U_{R}$ are reported in Table 5.8 .

\begin{tabular}{c|cccccc}
\hline & $U_{L}$ & $U_{1}$ & $U_{2}$ & $U_{3}$ & $U_{4}$ & $U_{R}$ \\
\hline$h$ & 0.5 & 0.262427 & 0.895486 & 0.922220 & 0.798666 & 1.0 \\
$u$ & 4.0 & 7.621172 & 2.233425 & 2.168679 & 2.168679 & 3.5 \\
$\theta$ & 3.0 & 3.0 & 3.0 & 3.0 & 4.0 & 4.0 \\
$a$ & 1.5 & 1.021888 & 1.021888 & 1.0 & 1.0 & 1.0 \\
\hline
\end{tabular}

Table 5.8: States that separate the elementary waves of the exact solution of the Riemann problem in Test 4: Construction A3.

One can see from the configuration of the exact solution 3.4 that the exact solution contains three waves propagating with the same zero speed. The errors of solution and orders of accuracy for Test 4 are reported in the Table 5.9 . 


\begin{tabular}{|c|c|c|}
\hline$N$ & $\left\|U_{h}-U\right\|_{L^{1}}$ & Order \\
\hline 100 & 0.1496 & \\
200 & 0.0972 & 0.62 \\
400 & 0.0612 & 0.67 \\
800 & 0.0391 & 0.65 \\
1600 & 0.0252 & 0.63 \\
\hline
\end{tabular}

Table 5.9: Errors of numerical approximations and orders of accuracy for different mesh sizes for Test 4 .

Figure 5.6 shows that the approximate solutions get closer to the exact solution when the mesh sizes get smaller. Notably, Table 5.9 shows that the errors become smaller and orders of accuracy are good as the mesh size gets smaller. So, Test 4 demonstrates the convergence of the approximate solutions by Godunov-type scheme to the exact solution when the initial data even for the resonant phenomenon, where several waves travel with a coinciding speed.
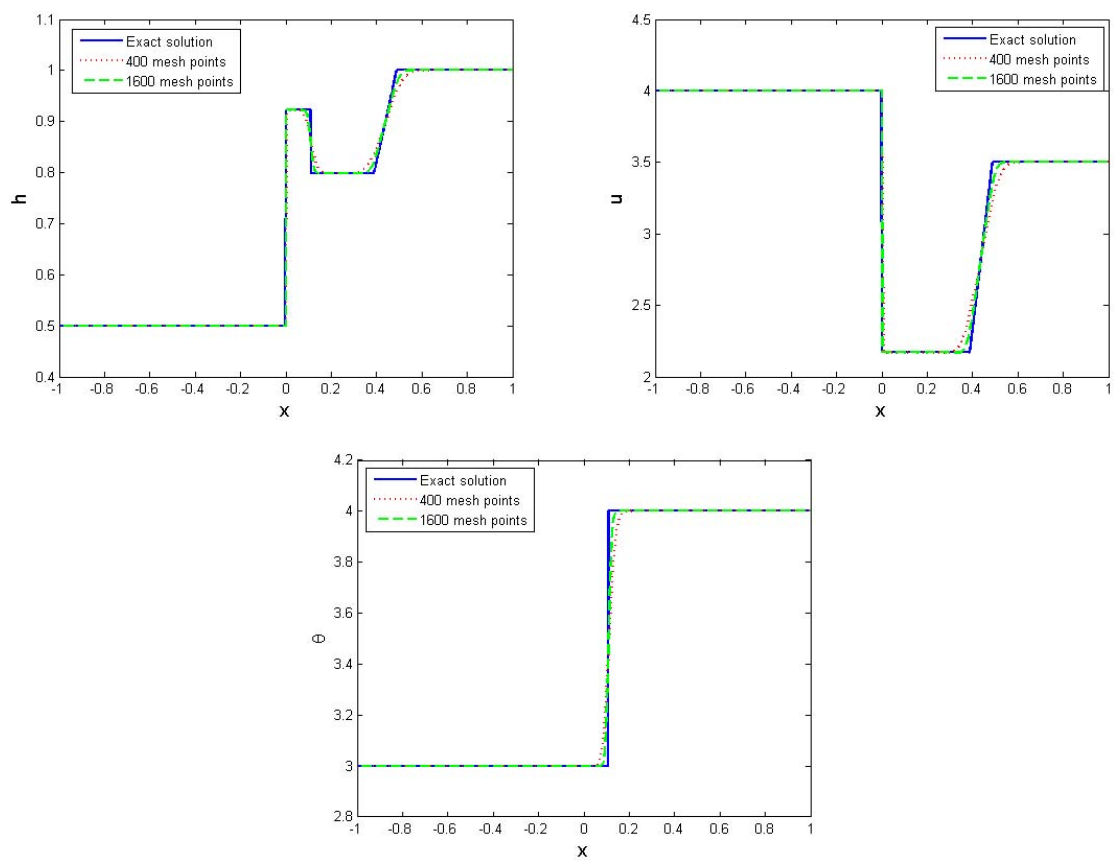

Figure 5.6: Exact solution and approximate solutions for different mesh sizes for Test 4 . 


\subsection{Test 5: Construction B1}

In this test we approximate a Riemann solution of Construction B1 with the Riemann data

$$
U_{0}(x)= \begin{cases}U_{L}=\left(h_{L}, u_{L}, \theta_{L}, a_{L}\right)=(0.5,2,4,1.5) \in G_{2} & \text { if } x<0, \\ U_{R}=\left(h_{R}, u_{R}, \theta_{R}, a_{R}\right)=(0.3,6.5,2,1) \in G_{1} & \text { if } x>0 .\end{cases}
$$

According to Construction B1, the exact solution is a 1-rarefaction wave from $U_{L}$ to $U_{1}$, followed by a stationary wave from $U_{1}$ to $U_{2}$, followed by a 1-shock wave from $U_{2}$ to $U_{3}$, followed by a 2-contact wave from $U_{3}$ to $U_{4}$, then followed by a 3 -shock wave from $U_{4}$ to $U_{R}$, where $U_{L}, U_{1}, U_{2}, U_{3}, U_{4}, U_{R}$ are reported in Table 5.10 .

\begin{tabular}{c|cccccc}
\hline & $U_{L}$ & $U_{1}$ & $U_{2}$ & $U_{3}$ & $U_{4}$ & $U_{R}$ \\
\hline$h$ & 0.5 & 0.333950 & 0.147735 & 0.240767 & 0.340497 & 0.3 \\
$u$ & 2.0 & 3.618124 & 8.178626 & 6.817442 & 6.817442 & 6.5 \\
$\theta$ & 4.0 & 4.0 & 4.0 & 4.0 & 2.0 & 2.0 \\
$a$ & 1.5 & 1.0 & 1.0 & 1.0 & 1.0 & 1.0 \\
\hline
\end{tabular}

Table 5.10: States that separate the elementary waves of the exact solution of the Riemann problem in Test 5: Construction B1.

The errors of solution and orders of accuracy for Test 5 are reported in Table 5.11 .

\begin{tabular}{|c|c|c|}
\hline$N$ & $\left\|U_{h}-U\right\|_{L^{1}}$ & Order \\
\hline 100 & 0.2869 & \\
200 & 0.1821 & 0.66 \\
400 & 0.1157 & 0.65 \\
800 & 0.0744 & 0.64 \\
1600 & 0.0477 & 0.64 \\
\hline
\end{tabular}

Table 5.11: Errors of numerical approximations and orders of accuracy for different mesh sizes for Test 5 .

Figure 5.7 shows that the approximate solutions get closer to the exact solution when the mesh sizes get smaller. Notably, Table 5.11 shows that the errors become smaller and orders of accuracy are good as the mesh size gets smaller. So, Test 5 demonstrates the convergence of the approximate solutions by Godunov-type scheme to the exact solution. 

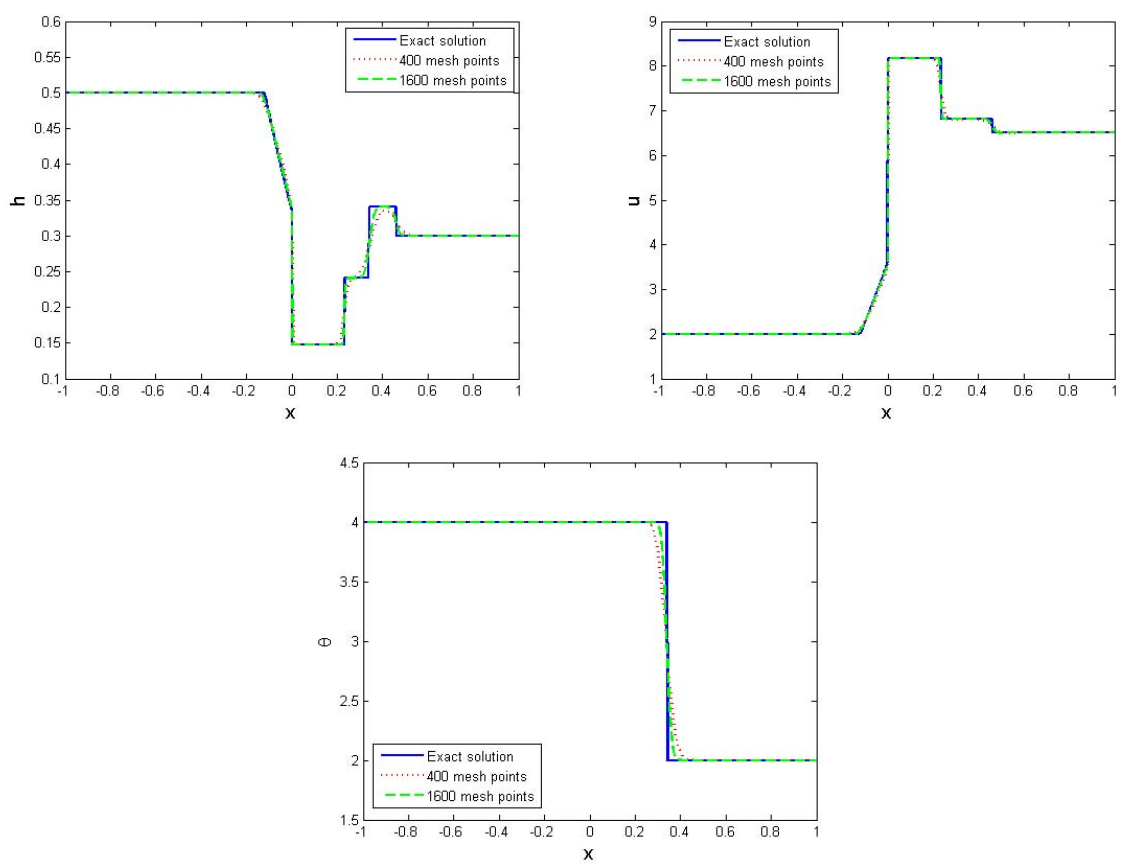

Figure 5.7: Exact solution and approximate solutions for different mesh sizes for Test 5 .

\subsection{Test 6: Construction B2}

In this test we approximate a Riemann solution of Construction B2 with the Riemann data

$$
U_{0}(x)= \begin{cases}U_{L}=\left(h_{L}, u_{L}, \theta_{L}, a_{L}\right)=(0.6,3,4,1.5) \in G_{2} & \text { if } x<0 \\ U_{R}=\left(h_{R}, u_{R}, \theta_{R}, a_{R}\right)=(1.5,2.4,3,1) \in G_{2} & \text { if } x>0\end{cases}
$$

\begin{tabular}{c|ccccc}
\hline & $U_{L}$ & $U_{1}$ & $U_{2}$ & $U_{3}$ & $U_{R}$ \\
\hline$h$ & 0.6 & 0.518204 & 1.156671 & 1.335608 & 1.5 \\
$u$ & 3.0 & 3.685363 & 1.651092 & 1.651092 & 2.4 \\
$\theta$ & 4.0 & 4.0 & 4.0 & 3.0 & 3.0 \\
$a$ & 1.5 & 1.5 & 1.0 & 1.0 & 1.0 \\
\hline
\end{tabular}

Table 5.12: States that separate the elementary waves of the exact solution of the Riemann problem in Test 6: Construction B2.

According to Construction B2, the exact solution is a 1-rarefaction wave from $U_{L}$ to $U_{1}$, followed by a stationary wave from $U_{1}$ to $U_{2}$, followed by a 2-contact wave from $U_{2}$ to 
$U_{3}$, then followed by a 3 -rarefaction wave from $U_{3}$ to $U_{R}$, where $U_{L}, U_{1}, U_{2}, U_{3}, U_{R}$ are reported in Table 5.12

The errors of solution and orders of accuracy for Test 6 are reported in Table 5.13.

\begin{tabular}{|c|c|c|}
\hline$N$ & $\left\|U_{h}-U\right\|_{L^{1}}$ & Order \\
\hline 100 & 0.1399 & \\
200 & 0.0906 & 0.63 \\
400 & 0.0579 & 0.65 \\
800 & 0.0374 & 0.63 \\
1600 & 0.0241 & 0.63 \\
\hline
\end{tabular}

Table 5.13: Errors of numerical approximations and orders of accuracy for different mesh sizes for Test 6 .

Figure 5.8 shows that the approximate solutions get closer to the exact solution when the mesh sizes get smaller. Notably, Table 5.13 shows that the errors become smaller and orders of accuracy are good as the mesh size gets smaller. So, Test 6 demonstrates the convergence of the approximate solutions by Godunov-type scheme to the exact solution.
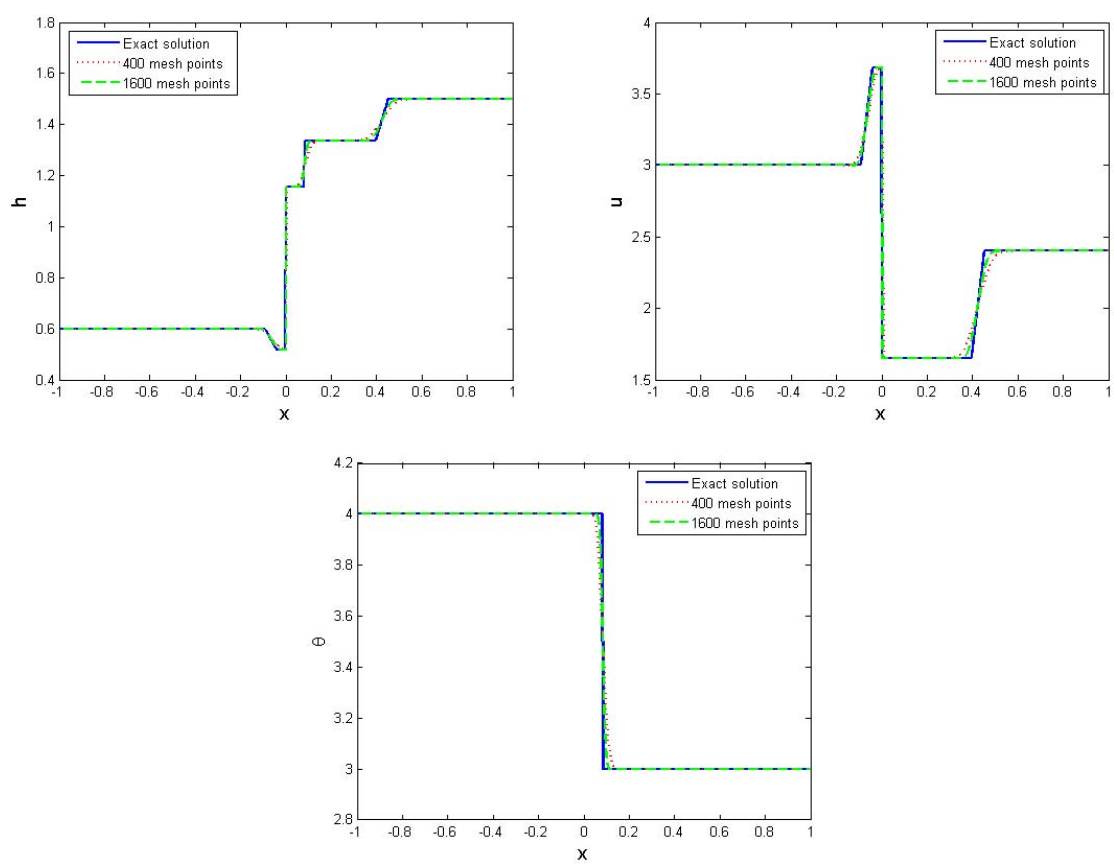

Figure 5.8: Exact solution and approximate solutions for different mesh sizes for Test 6 . 


\subsection{Test 7: A resonant phenomenon case (Construction B3)}

In this test we approximate a Riemann solution of Construction B3 with the Riemann data

$$
U_{0}(x)= \begin{cases}U_{L}=\left(h_{L}, u_{L}, \theta_{L}, a_{L}\right)=(0.5,2,4,1.5) \in G_{2} & \text { if } x<0 \\ U_{R}=\left(h_{R}, u_{R}, \theta_{R}, a_{R}\right)=(0.9,1.5,3,1) \in G_{2} & \text { if } x>0 .\end{cases}
$$

According to Construction B3, the exact solution is a 1-rarefaction wave from $U_{L}$ to $U_{1}$, followed by a stationary wave from $U_{1}$ to $U_{2}$, followed by a 1 -shock wave with zero-speed from $U_{2}$ to $U_{3}$, followed by a stationary wave $U_{3}$ to $U_{4}$, followed by a 2-contact wave from $U_{4}$ to $U_{5}$, then followed by a 3 -shock wave from $U_{5}$ to $U_{R}$, where $U_{L}, U_{1}, U_{2}, U_{3}, U_{4}, U_{5}$, $U_{R}$ are reported in Table 5.14 .

\begin{tabular}{c|ccccccc}
\hline & $U_{L}$ & $U_{1}$ & $U_{2}$ & $U_{3}$ & $U_{4}$ & $U_{5}$ & $U_{R}$ \\
\hline$h$ & 0.5 & 0.333950 & 0.168242 & 0.586556 & 0.785290 & 0.906775 & 0.9 \\
$u$ & 2.0 & 3.618124 & 7.181757 & 2.059946 & 1.538632 & 1.538632 & 1.5 \\
$\theta$ & 4.0 & 4.0 & 4.0 & 4.0 & 4.0 & 3.0 & 3.0 \\
$a$ & 1.5 & 1.5 & 1.174806 & 1.174806 & 1.0 & 1.0 & 1.0 \\
\hline
\end{tabular}

Table 5.14: States that separate the elementary waves of the exact solution of the Riemann problem in Test 7: Construction B3.

As we have seen by (3.7), the exact solution contains three waves propagating with the same zero speed. The errors of solution and orders of accuracy for Test 7 are reported in Table 5.15 .

\begin{tabular}{|c|c|c|}
\hline$N$ & $\left\|U_{h}-U\right\|_{L^{1}}$ & Order \\
\hline 100 & 0.1033 & \\
200 & 0.0670 & 0.62 \\
400 & 0.0425 & 0.66 \\
800 & 0.0269 & 0.66 \\
1600 & 0.0171 & 0.65 \\
\hline
\end{tabular}

Table 5.15: Errors of numerical approximations and orders of accuracy for different mesh sizes for Test 7 .

Figure 5.9 shows that the approximate solutions get closer to the exact solution when 
the mesh sizes get smaller. Notably, Table 5.15 shows that the errors become smaller and orders of accuracy are good as the mesh size gets smaller. So, Test 7 demonstrates the convergence of the approximate solutions by Godunov-type scheme to the exact solution in this very interesting resonant case, where the exact solutions contains several waves corresponding to different characteristic fields, which propagate with a coinciding shock speed.
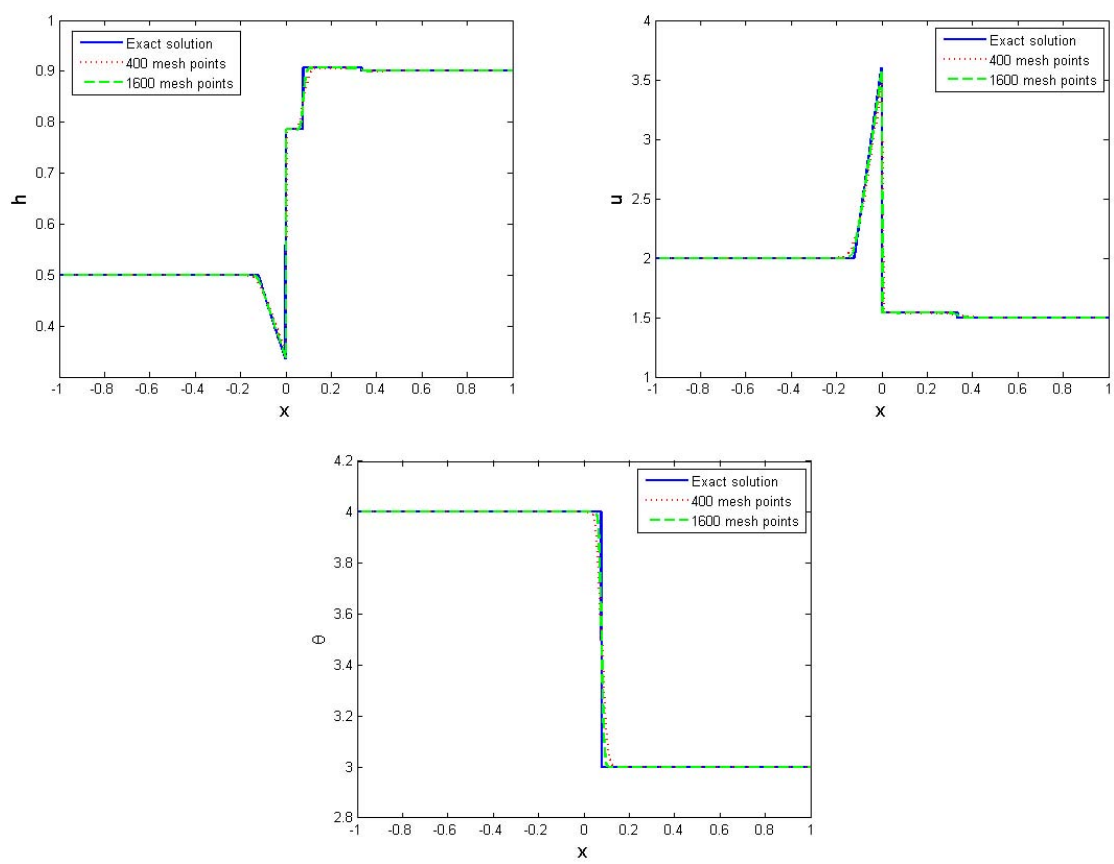

Figure 5.9: Exact solution and approximate solutions for different mesh sizes for Test 7 .

\subsection{Test 8: Wave interaction problem}

In this test we consider the Cauchy problem where the initial data consist of three constant states $U_{L}, U_{M}$ and $U_{R}$ with a jump discontinuity at two different points $x_{1}<x_{2}$, says, $x_{1}=0$ and $x_{2}=1$. At the beginning, the Cauchy problem can be seen as two separated Riemann problems centered at $x_{1}$ and $x_{2}$. After a certain time $t=t_{1}$, the wave with highest speed in the solution of the Riemann problem centered at $x_{1}$ interacts with the wave with lowest speed in the solution of the Riemann problem centered at $x_{2}$. New waves will be generated after the wave interaction at $t_{1}$. We will calculate the time of wave interaction $t_{1}$ and will compute the approximate solution by the scheme after this time and compare with the exact solution. It is interesting to see the interaction of waves where the bottom level suffers a jump. Therefore, we will consider the interaction of a 3 -shock starting from $x_{1}$ with a 4-stationary contact discontinuity along $x_{2}$. This two 
waves meet at $(x, t)=\left(x_{2}, t_{1}\right)$. New waves are generated from this wave interaction, and they are merely elementary waves of the local Riemann problem centered at $\left(x_{2}, t_{1}\right)$.

Precisely, we consider the Cauchy problem with the following initial condition

$$
U_{0}(x)= \begin{cases}U_{L}=\left(h_{L}, u_{L}, \theta_{L}, a_{L}\right)=(0.5,4,3,1.5) \in G_{1} & \text { if } x<0 \\ U_{M}=\left(h_{M}, u_{M}, \theta_{M}, a_{M}\right)=(0.3,3.5,4,1) \in G_{1} & \text { if } 0<x<1 \\ U_{R}=\left(h_{R}, u_{R}, \theta_{R}, a_{R}\right)=(0.152633,6.879229,4,0.7) \in G_{1} & \text { if } x>1\end{cases}
$$

see Figure 5.10 .
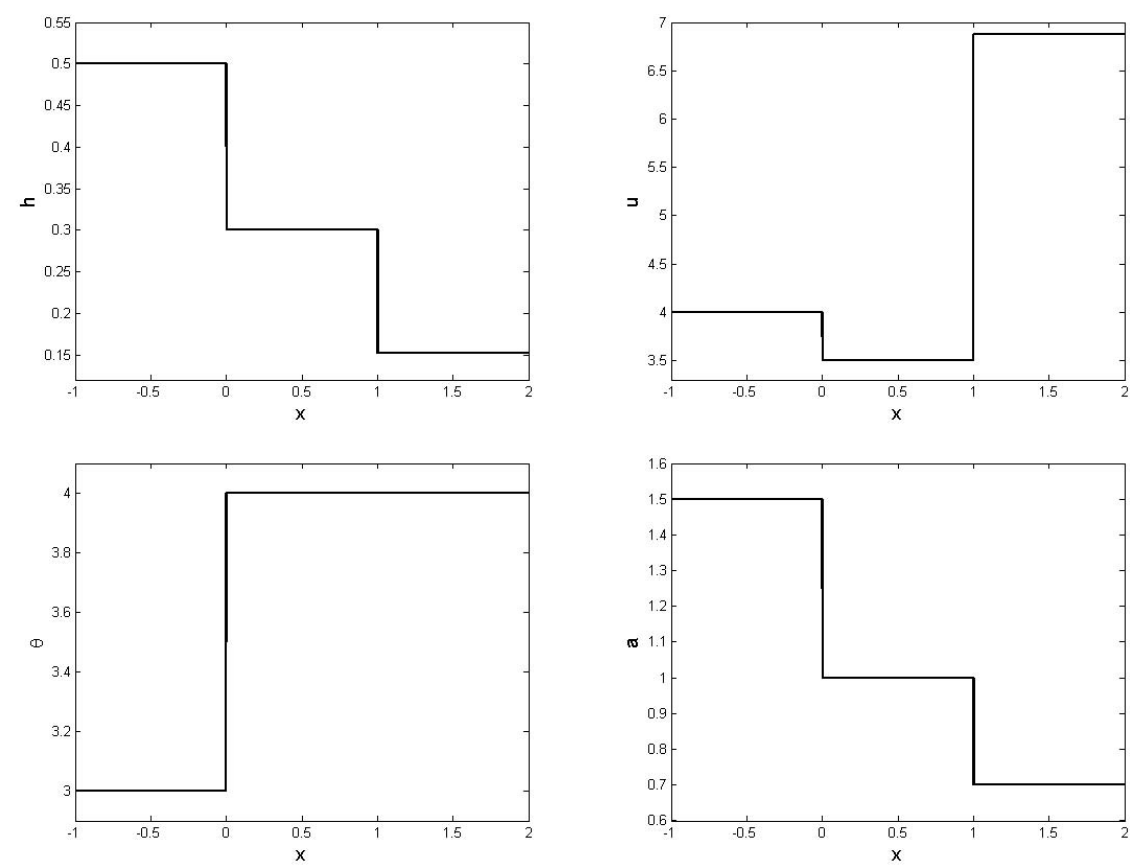

Figure 5.10: The initial condition (5.1).

It holds that the Riemann data $\left(U_{L}, U_{M}\right)$ at $x=0$ satisfies Construction A1 and the Riemann data $\left(U_{M}, U_{R}\right)$ at $x=1$ satisfies (2.11). So, while $t<t_{1}$, the Riemann solution at $x=0$ is

$$
W_{4}\left(U_{L}, U_{1}\right) \oplus S_{1}\left(U_{1}, U_{2}\right) \oplus W_{2}\left(U_{2}, U_{3}\right) \oplus S_{3}\left(U_{3}, U_{M}\right),
$$

and the Riemann solution at $x=1$ is just the 4 -contact stationary wave

$$
W_{4}\left(U_{M}, U_{R}\right)
$$

see Figure 5.11. A wave interaction occurs when the 3 -shock wave $S_{3}\left(U_{3}, U_{M}\right)$ from $x=0$ meets the 4-stationary contact wave $W_{4}\left(U_{M}, U_{R}\right)$ at $\left(x=1, t_{1}\right)$. The time $t_{1}$ is determined 
by equating

$$
x=\sigma_{3}\left(U_{3}, U_{R}\right) t=1, \quad \text { or } \quad t_{1} \approx 0.1204
$$

Observe that the 2-contact $W_{2}\left(U_{2}, U_{3}\right)$ starting at $x=0$ may meet the 4-contact $W_{4}\left(U_{M}\right.$, $\left.U_{R}\right)$ at a larger time $t_{2}$, when the line $x=\sigma_{2}\left(U_{2}, U_{3}\right) t$ cuts the line $x=1$. It is easy to check that $t_{2} \approx 0.1927$.

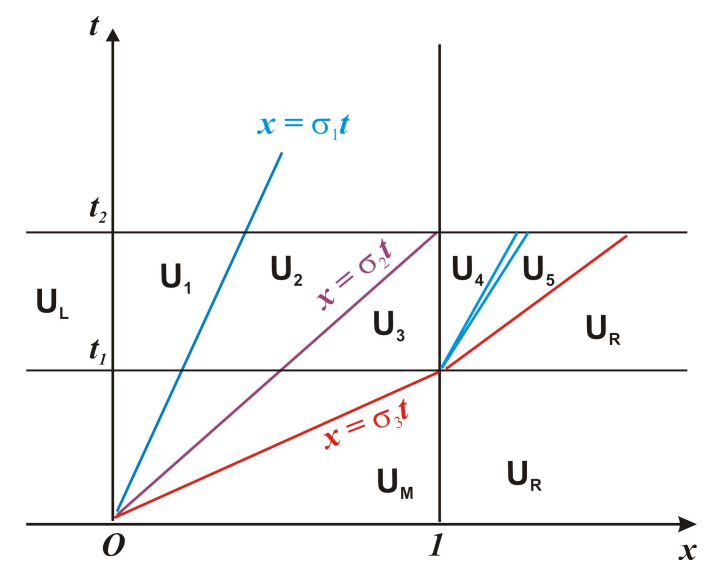

Figure 5.11: The exact solution for Test 8 at time $t_{1}<t<t_{2}$.

We will conduct the test at a time $t_{*}$ between these two values $t_{1}$ and $t_{2}$. For example, we take

$$
t_{*}=0.15
$$

Note that after the first wave interaction between the 3 -shock wave $S_{3}\left(U_{3}, U_{M}\right)$ and the 4-stationary contact wave $W_{4}\left(U_{M}, U_{R}\right)$, new waves are generated and form a solution of the local Riemann problem centered at $\left(x=1, t_{1}\right)$ with the with data $\left(U_{3}, U_{R}\right)$. It is easy to see that after the first wave interaction and before the second wave interaction, that is,

$$
t_{1}<t<t_{2}
$$

the exact solution of this local Riemann problem centered at $\left(x=1, t_{1}\right)$ with the initial data $\left(U_{3}, U_{R}\right)$ is given by

$$
W_{4}\left(U_{3}, U_{4}\right) \oplus R_{1}\left(U_{4}, U_{5}\right) \oplus S_{3}\left(U_{5}, U_{R}\right)
$$

Overall, the exact solution for (1.1) with initial data (5.1) at the time $t=0.15$ is

$$
W_{4}\left(U_{L}, U_{1}\right) \oplus S_{1}\left(U_{1}, U_{2}\right) \oplus W_{2}\left(U_{2}, U_{3}\right) \oplus W_{4}\left(U_{3}, U_{4}\right) \oplus R_{1}\left(U_{4}, U_{5}\right) \oplus S_{3}\left(U_{5}, U_{R}\right),
$$

see Figure 5.11, where the states $U_{1}, U_{2}, U_{3}, U_{4}$ and $U_{5}$ are reported in Table 5.16 . 


\begin{tabular}{c|ccccc}
\hline & $U_{1}$ & $U_{2}$ & $U_{3}$ & $U_{4}$ & $U_{5}$ \\
\hline$h$ & 0.259147 & 0.534519 & 0.462907 & 0.302650 & 0.261204 \\
$u$ & 7.717632 & 5.190415 & 5.190415 & 7.938793 & 8.427843 \\
$\theta$ & 3.0 & 3.0 & 4.0 & 4.0 & 4.0 \\
$a$ & 1.0 & 1.0 & 1.0 & 0.7 & 0.7 \\
\hline
\end{tabular}

Table 5.16: States that separate the elementary waves of the exact solution of the wave interaction problem in Test 8.

\begin{tabular}{|c|c|c|}
\hline$N$ & $\left\|U_{h}-U\right\|_{L^{1}}$ & Order \\
\hline 100 & 0.3577 & \\
200 & 0.2184 & 0.71 \\
400 & 0.1337 & 0.71 \\
800 & 0.0834 & 0.68 \\
1600 & 0.0504 & 0.73 \\
\hline
\end{tabular}

Table 5.17: Errors of numerical approximations and orders of accuracy for different mesh sizes for Test 8 .
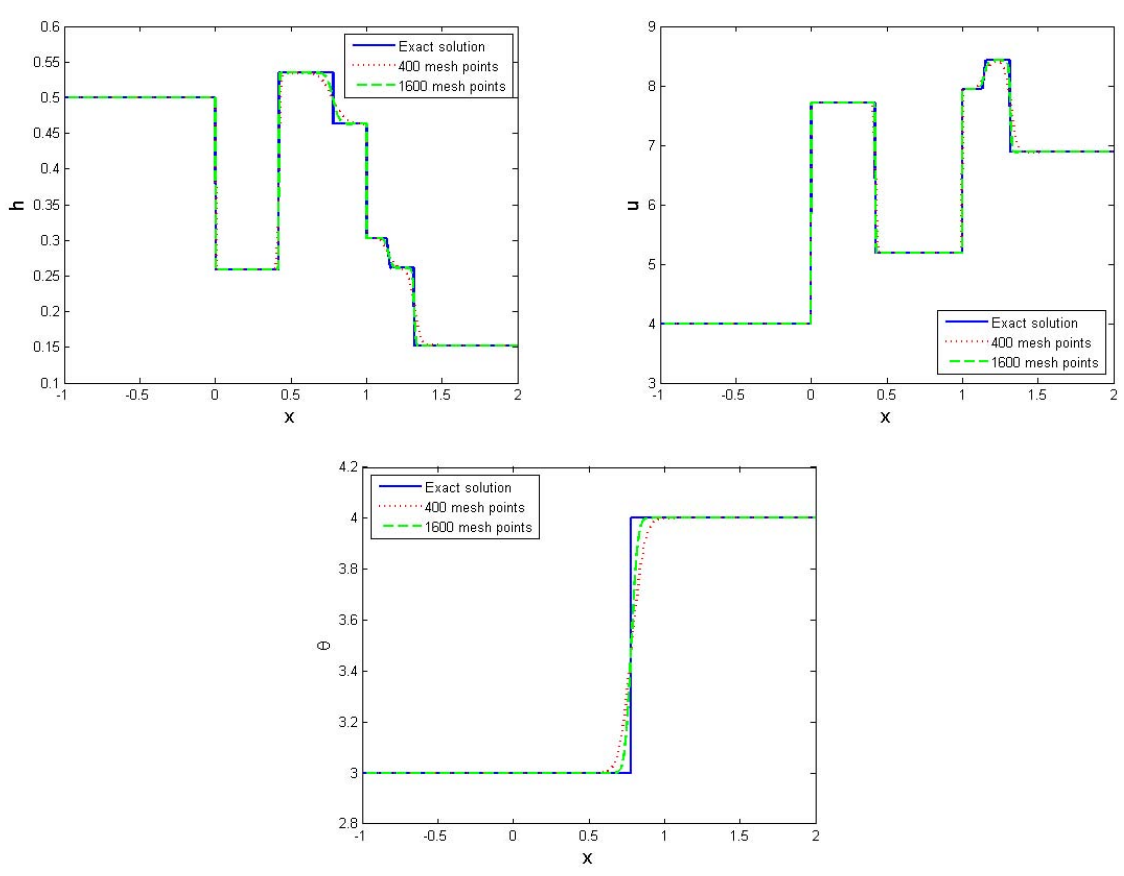

Figure 5.12: Approximations by the Godunov-type scheme at the time $t_{*}=0.15$ after the time waves interact $t_{1} \approx 0.1204$ for Test 8 . 
The errors of solution and orders of accuracy for Test 8 are reported in Table 5.17 .

Figure 5.12 shows that the approximate solutions get closer to the exact solution when the mesh sizes get smaller. Table 5.17] shows that the errors become smaller and orders of accuracy are good as the mesh size gets smaller. So, Test 8 demonstrates the convergence of the approximate solutions by Godunov-type scheme to the exact solution.

\section{Conclusions}

The fact that the Ripa system (1.1) is nonconservative makes it hard for existing schemes. Therefore, the study of numerical approximations for solutions of the system has been a very challenging issue. Here, we aim to employ the idea of the Godunov scheme for conservation laws by using exact solutions of the local Riemann problem. For this purpose, we first present exact solutions of the Riemann problem for $\sqrt{1.1}-(1.2)$ in a computational form such that the states determining elementary waves can be computed from the nonlinear algebraic equations. In particular, this provides us with the exact Riemann solvers, which can be used to build up a Godunov-type scheme. Interesting properties of this scheme are established, where we show that the scheme is well-balanced, and it preserves the positivity of the water height. The accuracy is computed for all the numerical tests, which shows the convergence of the scheme where the initial data belong to the supercritical region, or subcritical region, or both. Especially, approximate solutions by our scheme still converge to the exact solutions even in the resonant phenomenon, where the exact solutions contain several distinct waves propagating with the same shock speed. Furthermore, the approximations by the Godunov-type scheme of the solution of the wave interaction problem are considered and give very suitable results.

\section{Acknowledgments}

The authors would like to thank the reviewers for their very valuable comments and suggestions. This research is funded by Vietnam National University HoChiMinh City (VNU-HCM) under grant number B2018-28-01.

\section{References}

[1] A. Ambroso, C. Chalons, F. Coquel and T. Galié, Relaxation and numerical approximation of a two-fluid two-pressure diphasic model, M2AN Math. Model. Numer. Anal. 43 (2009), no. 6, 1063-1097.

[2] A. Ambroso, C. Chalons and P.-A. Raviart, A Godunov-type method for the sevenequation model of compressible two-phase flow, Comput. \& Fluids 54 (2012), 67-91. 
[3] E. Audusse, F. Bouchut, M.-O. Bristeau, R. Klein and B. Perthame, A fast and stable well-balanced scheme with hydrostatic reconstruction for shallow water flows, SIAM J. Sci. Comput. 25 (2004), no. 6, 2050-2065.

[4] M. Baudin, F. Coquel and Q.-H. Tran, A semi-implicit relaxation scheme for modeling two-phase flow in a pipeline, SIAM J. Sci. Comput. 27 (2005), no. 3, 914-936.

[5] R. Botchorishvili, B. Perthame and A. Vasseur, Equilibrium schemes for scalar conservation laws with stiff sources, Math. Comp. 72 (2003), no. 241, 131-157.

[6] R. Botchorishvili and O. Pironneau, Finite volume schemes with equilibrium type discretization of source terms for scalar conservation laws, J. Comput. Phys. 187 (2003), no. 2, 391-427.

[7] A. Chertock, A. Kurganov and Y. Liu, Central-upwind schemes for the system of shallow water equations with horizontal temperature gradients, Numer. Math. 127 (2014), no. 4, 595-639.

[8] A. Chinnayya, A.-Y. LeRoux and N. Seguin, A well-balanced numerical scheme for the approximation of the shallow-water equations with topography: the resonance phenomenon, Int. J. Finite 1 (2004), no. 1, 33 pp.

[9] F. Coquel, J.-M. Hérard, K. Saleh and N. Seguin, Two properties of two-velocity twopressure models for two-phase flows, Commun. Math. Sci. 12 (2014), no. 3, 593-600.

[10] D. H, Cuong and M. D. Thanh, A Godunov-type scheme for the isentropic model of a fluid flow in a nozzle with variable cross-section, Appl. Math. Comput. 256 (2015), 602-629.

[11] G. Dal Maso, P. G. Lefloch and F. Murat, Definition and weak stability of nonconservative products, J. Math. Pures Appl. (9) 74 (1995), no. 6, 483-548.

[12] J. M. Gallardo, C. Parés and M. Castro, On a well-balanced high-order finite volume scheme for shallow water equations with topography and dry areas, J. Comput. Phys. 227 (2007), no. 1, 574-601.

[13] T. Gallouët, J.-M. Hérard and N. Seguin, Some approximate Godunov schemes to compute shallow-water equations with topography, Comput. \& Fluids 32 (2003), no. 4, 479-513.

[14] J. M. Greenberg and A. Y. Leroux, A well-balanced scheme for the numerical processing of source terms in hyperbolic equations, SIAM J. Numer. Anal. 33 (1996), no. 1, $1-16$. 
[15] X. Han and G. Li, Well-balanced finite difference WENO schemes for the Ripa model, Comput. \& Fluids 134-135 (2016), 1-10.

[16] T. Y. Hou and P. G. LeFloch, Why nonconservative schemes converge to wrong solutions: error analysis, Math. Comp. 62 (1994), no. 206, 497-530.

[17] E. Isaacson and B. Temple, Convergence of the $2 \times 2$ Godunov method for a general resonant nonlinear balance law, SIAM J. Appl. Math. 55 (1995), no. 3, 625-640.

[18] D. Kröner and M. D. Thanh, Numerical solutions to compressible flows in a nozzle with variable cross-section, SIAM J. Numer. Anal. 43 (2005), no. 2, 796-824.

[19] P. G. LeFloch and M. D. Thanh, A Godunov-type method for the shallow water equations with discontinuous topography in the resonant regime, J. Comput. Phys. 230 (2011), no. 20, 7631-7660.

[20] G. Li, V. Caleffi and Z. Qi, A well-balanced finite difference WENO scheme for shallow water flow model, Appl. Math. Comput. 265 (2015), 1-16.

[21] G. Li, L. Song and J. Gao, High order well-balanced discontinuous Galerkin methods based on hydrostatic reconstruction for shallow water equations, J. Comput. Appl. Math. 340 (2018), 546-560.

[22] S. Qian, F. Shao and G. Li, High order well-balanced discontinuous Galerkin methods for shallow water flow under temperature fields, Comput. Appl. Math. 37 (2018), no. $5,5775-5794$.

[23] P. Ripa, Conservation laws for primitive equations models with inhomogeneous layers, Geophys. Astrophys. Fluid Dynam. 70 (1993), no. 1-4, 85-111.

[24] _ On improving a one-layer ocean model with thermodynamics, J. Fluid Mech. 303 (1995), 169-201.

[25] G. Rosatti and L. Begnudelli, The Riemann problem for the one-dimensional, freesurface shallow water equations with a bed step: theoretical analysis and numerical simulations, J. Comput. Phys. 229 (2010), no. 3, 760-787.

[26] C. Sánchez-Linares, T. Morales de Luna and M. J. Castro Diaz, A HLLC scheme for Ripa model, Appl. Math. Comput. 72 (2016), 369-384.

[27] R. Saurel and R. Abgrall, A multiphase Godunov method for compressible multifluid and multiphase flows, J. Comput. Phys. 150 (1999), no. 2, 425-467. 
[28] B. Tian, E. F. Toro and C. E. Castro, A path-conservative method for a five-equation model of two-phase flow with an HLLC-type Riemann solver, Comput. \& Fluids 46 (2011), 122-132.

[29] M. D. Thanh, The Riemann problem for a nonisentropic fluid in a nozzle with discontinuous cross-sectional area, SIAM J. Appl. Math. 69 (2009), no. 6, 1501-1519.

[30] _ A phase decomposition approach and the Riemann problem for a model of two-phase flows, J. Math. Anal. Appl. 418 (2014), no. 2, 569-594.

[31] _ The Riemann problem for the shallow water equations with horizontal temperature gradients, Appl. Math. Comput. 325 (2018), 159-178.

[32] M. D. Thanh and N. X. Thanh, Well-balanced numerical schemes for shallow water equations with horizontal temperature gradient, Accepted in Bull. Malays. Math. Sci. Soc. (2018).

[33] R. Touma and C. Klingenberg, Well-balanced central finite volume methods for the Ripa system, Appl. Num. Math. 97 (2015), 42-68.

Nguyen Xuan Thanh

Department of Mathematics and Computer Science, University of Science, Vietnam National University - Ho Chi Minh City, 227 Nguyen Van Cu str., District 5, Ho Chi Minh City, Vietnam

E-mail address: mr.nxthanh@gmail.com

Mai Duc Thanh

Department of Mathematics, International University, Vietnam National University - Ho Chi Minh City, Quarter 6, Linh Trung Ward, Thu Duc District, Ho Chi Minh City, Vietnam

E-mail address: mdthanh@hcmiu.edu.vn

Dao Huy Cuong

Department of Mathematics, Ho Chi Minh City University of Education, 280 An Duong Vuong str., District 5, Ho Chi Minh City, Vietnam

E-mail address: cuongdh@hcmue.edu.vn, cuongnhc82@gmail.com 